\title{
EMBEDDABILITY INTO RELATIONAL LATTICES IS UNDECIDABLE
}

\author{
LUIGI SANTOCANALE
}

\begin{abstract}
The natural join and the inner union operations combine relations of a database. Tropashko and Spight realized that these two operations are the meet and join operations in a class of lattices, known by now as the relational lattices. They proposed then lattice theory as an algebraic approach to the theory of databases alternative to the relational algebra. Litak et al. proposed an axiomatization of relational lattices over the signature that extends the pure lattice signature with a constant and argued that the quasiequational theory of relational lattices over this extended signature is undecidable.

We prove in this paper that embeddability is undecidable for relational lattices. More precisely, it is undecidable whether a finite subdirectly-irreducible lattice can be embedded into a relational lattice. Our proof is a reduction from the coverability problem of a multimodal frame by a universal product frame and, indirectly, from the representability problem for relation algebras.

As corollaries we obtain the following results: the quasiequational theory of relational lattices over the pure lattice signature is undecidable and has no finite base; there is a quasiequation over the pure lattice signature which holds in all the finite relational lattices but fails in an infinite relational lattice.
\end{abstract}

\section{INTRODUCTION}

The natural join and the inner union operations combine relations (i.e. tables) of a database. Most of today's web programs query their databases making repeated use of the natural join and of the union, of which the inner union is a mathematically well behaved variant. Tropashko and Spight realized [26, 25] that these two operations are the meet and join operations in a class of lattices, known by now as the class of relational lattices. They proposed then lattice theory as an algebraic approach, alternative to Codd's relational algebra 4, to the theory of databases.

An important first attempt to axiomatize these lattices is due to Litak, Mikulás, and Hidders [16. These authors propose an axiomatization, comprising equations and quasiequations, in a signature that extends the pure lattice signature with a constant, the header constant. A main result of that paper is that the quasiequational theory of relational lattices is undecidable in this extended signature. Their proof mimics Maddux's proof that the equational theory of cylindric algebras of dimension $n \geq 3$ is undecidable [17.

We have investigated in 23 equational axiomatizations for relational lattices using as tool the duality theory for finite lattices developed in 22. A conceptual contribution from 23] is to make explicit the similarity between the developing theory of relational lattices and the well established theory of combination of modal logics, see e.g. [14. This was achieved on the syntactic side, but also on the semantic side, by identifying some key properties of the structures dual to the finite atomistic lattices in the variety generated by the relational lattices, see [23, Theorem 7]. These 
properties make the dual structures into frames for commutator multimodal logics in a natural way.

In this paper we exploit this similarity to transfer results from the theory of multidimensional modal logics to lattice theory. Our main result is that it is undecidable whether a finite subdirectly irreducible lattice can be embedded into a relational lattice. We prove this statement by reducing to it the coverability problem of a frame by a universal $\mathrm{S} 5^{3}$-product frame, a problem shown to be undecidable in [12. As stated there, the coverability problem is - in light of standard duality theory - a direct reformulation of the representability problem of finite simple relation algebras, problem shown to be undecidable by Hirsch and Hodkinson [11.

Our main result and its proof allow us to derive further consequences. Firstly, we refine the undecidability theorem of [16] and prove that the quasiequational theory of relational lattices in the pure lattice signature is undecidable as well and has no finite base. Then we argue that there is a quasiequation that holds in all the finite relational lattices, but fails in an infinite one. For the latter result, we rely on the work by Hirsch, Hodkinson, and Kurucz 12 who constructed a finite 3-multimodal frame which has no finite $p$-morphism from a finite universal $\mathrm{S} 5^{3}$-product frame, but has a $p$-morphism from an infinite one. On the methodological side, we wish to point out our use of generalized ultrametric spaces to tackle these problems. A key idea in the proof of the main result is the characterization of universal S5 ${ }^{A}$-product frames as pairwise complete generalized ultrametric spaces with distance valued in the Boolean algebra $P(A)$, a characterization that holds when $A$ is finite.

The paper is structured as follows. We recall in Section 2 few definitions and facts on frames and lattices. Relational lattices are introduced in Section 3 In Section 4 we give an outline of the proof of our main technical result - the undecidability of embeddability of a finite subdirectly-irreducible lattice into a relational lattice - and derive from it the other results. In Section 5 we show how to construct a lattice from a frame and use functoriality of this construction to argue that such lattice embeds into a relational lattice whenever the frame is a $p$-morphic image of a universal product frame. The proof of the converse statement is carried out in Section 8 The technical tools needed to prove the converse are developed Sections 6 and 7 The theory of generalized ultrametric spaces over a powerset Boolean algebra and the aforementioned characterization of $\mathrm{S} 5^{A}$-product frames as pairwise complete spaces over $P(A)$ appear in Section 6 . In Section 7 we study embeddings of finite subdirectly-irreducible lattices into relational lattices and prove that we can assume that these embeddings preserve bounds. This task is needed so to exclude the constants $\perp$ and $T$ (denoting the bounds) from the signature of lattice theory.

\section{Frames AND LATTICES}

Frames. Let $A$ be a set of actions. An A-multimodal frame (briefly, an $A$-frame or a frame) is a structure $\mathfrak{F}=\left\langle X_{\mathfrak{F}},\left\{R_{a} \mid a \in A\right\}\right\rangle$ where, for each $a \in A, R_{a}$ is a binary relation on $X_{\mathfrak{F}}$. We say that an $A$-frame is $\mathrm{S} 4$ if each $R_{a}$ is reflexive and transitive. If $\mathfrak{F}_{0}$ and $\mathfrak{F}_{1}$ are two $A$-frames, then a -morphism from $\mathfrak{F}_{0}$ to $\mathfrak{F}_{1}$ is a function $\psi: X_{\mathfrak{F}_{0}} \longrightarrow X_{\mathfrak{F}_{1}}$ such that, for each $a \in A$,

- if $x R_{a} y$, then $\psi(x) R_{a} \psi(y)$,

- if $\psi(x) R_{a} z$, then $x R_{a} y$ for some $y$ with $\psi(y)=z$.

Let us mention that $A$-multimodal frames and $p$-morphisms form a category. 
A frame $\mathfrak{F}$ is said to be rooted (or initial, see [21]) if there is $f_{0} \in X_{\mathfrak{F}}$ such that every other $f \in X_{\mathfrak{F}}$ is reachable from $f_{0}$. We say that an $A$-frame $\mathfrak{F}$ is full if, for each $a \in A$, there exists $f, g \in X_{\mathfrak{F}}$ such that $f \neq g$ and $f R_{a} g$. If $G=(V, D)$ is a directed graph, then we shall say that $G$ is rooted if it is rooted as a unimodal frame.

A particular class of frames we shall deal with are the universal $\mathrm{S}^{A}$-product frames. These are the frames $\mathfrak{U}$ with $X_{\mathfrak{U}}=\prod_{a \in A} X_{a}$ and $x R_{a} y$ if and only if $x_{i}=y_{i}$ for each $i \neq a$, where $x:=\left\langle x_{i} \mid i \in A\right\rangle$ and $y:=\left\langle y_{i} \mid i \in A\right\rangle$.

Let $\alpha \subseteq A, \mathfrak{F}$ be an $A$-frame, $x, y \in X_{\mathfrak{F}}$. An $\alpha$-path from $x$ to $y$ is a sequence $x=x_{0} R_{a_{0}} x_{1} \ldots x_{k-1} R_{a_{k-1}} x_{k}=y$ with $\left\{a_{0}, \ldots, a_{k-1}\right\} \subseteq \alpha$. We use then the notation $x \stackrel{\alpha}{\rightarrow} y$ to mean that there is an $\alpha$-path from $x$ to $y$. Notice that if $\mathfrak{F}$ is an S4 $A$-frame, then $x \stackrel{\{a\}}{\longrightarrow} y$ if and only if $x R_{a} y$.

Orders and lattices. We assume some basic knowledge of order and lattice theory as presented in standard monographs 5, 9. Most of the tools we use in this paper originate from the monograph [8] and have been further developed in [22].

A lattice is a poset $L$ such that every finite non-empty subset $X \subseteq L$ admits a smallest upper bound $\bigvee X$ and a greatest lower bound $\bigwedge X$. A lattice can also be understood as a structure $\mathfrak{A}$ for the functional signature $(\vee, \wedge)$, such that the interpretations of these two binary function symbols both give $\mathfrak{A}$ the structure of an idempotent commutative semigroup, the two semigroup structures being connected by the absorption laws $x \wedge(y \vee x)=x$ and $x \vee(y \wedge x)=x$. Once a lattice is presented as such structure, the order is recovered by stating that $x \leq y$ holds if and only if $x \wedge y=x$.

A lattice $L$ is complete if any subset $X \subseteq L$ admits a smallest upper bound $\bigvee X$. It can be shown that this condition implies that any subset $X \subseteq L$ admits a greatest lower bound $\bigwedge X$. A lattice is bounded if it has a least element $\perp$ and a greatest element $T$. A complete lattice (in particular, a finite lattice) is bounded, since $\bigvee \emptyset$ and $\wedge \emptyset$ are, respectively, the least and greatest elements of the lattice.

If $P$ and $Q$ are partially ordered sets, then a function $f: P \longrightarrow Q$ is orderpreserving (or monotone) if $p \leq p^{\prime}$ implies $f(p) \leq f\left(p^{\prime}\right)$. If $L$ and $M$ are lattices, then a function $f: L \longrightarrow M$ is a lattice morphism if it preserves the lattice operations $\vee$ and $\wedge$. A lattice morphism is always order-preserving. A lattice morphism $f: L \longrightarrow M$ between bounded lattices $L$ and $M$ is bound-preserving if $f(\perp)=\perp$ and $f(\top)=\top$. A function $g: Q \longrightarrow P$ is said to be left adjoint to an order-preserving $f: P \longrightarrow Q$ if $g(q) \leq p$ holds if and only if $q \leq f(p)$ holds; such a left adjoint, when it exists, is unique. If $L$ is finite, $M$ is bounded, and $f: L \longrightarrow M$ is a bound-preserving lattice morphism, then a left adjoint to $f$ always exists and preserves the constant $\perp$ and the operation $\vee$.

A Moore family on a set $U$ is a collection $\mathcal{F}$ of subsets of $U$ which is closed under arbitrary intersections. Given a Moore family $\mathcal{F}$ on $U$, the correspondence sending $Z \subseteq U$ to $\bar{Z}:=\bigcap\{Y \in \mathcal{F} \mid Z \subseteq Y\}$ is a closure operator on $U$, that is, an order-preserving inflationary and idempotent endofunction of $P(U)$. The subsets in $\mathcal{F}$, called the closed sets, are exactly the fixpoints of this closure operator. We can give $\mathcal{F}$ a lattice structure by defining

$$
\bigwedge x:=\bigcap x, \quad \bigvee x:=\overline{\bigcup x}
$$


Let $L$ be a complete lattice. An element $j \in L$ is completely join-irreducible if $j=\bigvee X$ implies $j \in X$, for each $X \subseteq L$; the set of completely join-irreducible elements of $L$ is denoted here $\mathcal{J}_{c}(L)$. A complete lattice is spatial if every element is the join of the completely join-irreducible elements below it. An element $j \in \mathcal{J}_{c}(L)$ is said to be join-prime if $j \leq \bigvee X$ implies $j \leq x$ for some $x \in X$, for each finite subset $X$ of $L$. If $x$ is not join-prime, then we say that $x$ is non-join-prime. An atom of a lattice $L$ is an element of $L$ such that $\perp$ is the only element strictly below it. A spatial lattice is atomistic if every element of $\mathcal{J}_{\mathrm{c}}(L)$ is an atom.

For $j \in \mathcal{J}_{\mathrm{c}}(L)$, a join-cover of $j$ is a subset $X \subseteq L$ such that $j \leq \bigvee X$. For $X, Y \subseteq L$, we say that $X$ refines $Y$, and write $X \ll Y$, if for all $x \in X$ there exists $y \in Y$ such that $x \leq y$. A join-cover $X$ of $j$ is said to be minimal if $j \leq \bigvee Y$ and $Y \ll X$ implies $X \subseteq Y$; we write $j \triangleleft_{\mathrm{m}} X$ if $X$ is a minimal join-cover of $j$. In a spatial lattice, if $j \triangleleft_{\mathrm{m}} X$, then $X \subseteq \mathcal{J}_{\mathrm{c}}(L)$. If $j \triangleleft_{\mathrm{m}} X$, then we say that $X$ is a non-trivial minimal join-cover of $j$ if $X \neq\{j\}$. The word perfect is used in the lattice-theoretic literature with different meanings [6, 2]. We use here something different:

Definition 1. We say that a complete lattice is pluperfect if it is spatial and for each $j \in \mathcal{J}_{\mathrm{c}}(L)$ and $X \subseteq L$, if $j \leq \bigvee X$, then $Y \ll X$ for some $Y$ such that $j \triangleleft_{\mathrm{m}} Y$. The OD-graph of a pluperfect lattice $L$ is the structure $\left\langle\mathcal{J}_{\mathrm{c}}(L), \leq, \triangleleft_{\mathrm{m}}\right\rangle$.

That is, in a pluperfect lattice every cover refines to a minimal one. With respect to analogous definitions, such as that of a lattice with the $\Sigma$-minimal join-cover refinement property [27] or that of a strongly spatial lattice [24, we do not require here that the set $Y$ in the relation $j \triangleleft_{\mathrm{m}} Y$ is finite, nor that, for a given $j$, there are a finite number of these sets.

Notice that every finite lattice is pluperfect. If $L$ is a pluperfect lattice, then we say that $X \subseteq \mathcal{J}_{\mathrm{c}}(L)$ is closed if it is a downset and $j \triangleleft_{\mathrm{m}} C \subseteq X$ implies $j \in X$. Closed subsets of $\mathcal{J}_{\mathrm{c}}(L)$ form a Moore family. The interest of considering pluperfect lattices stems from the following representation theorem stated in [19] for finite lattices; its generalization to pluperfect lattices is straightforward.

Theorem 2. Let $L$ be a pluperfect lattice and let $\mathrm{L}\left(\mathcal{J}_{\mathrm{c}}(L), \leq, \triangleleft_{\mathrm{m}}\right)$ be the lattice of closed subsets of $\mathcal{J}_{\mathrm{c}}(L)$. The mapping $l \mapsto\left\{j \in \mathcal{J}_{\mathrm{c}}(L) \mid j \leq l\right\}$ is a lattice isomorphism from $L$ to $\mathrm{L}\left(\mathcal{J}_{\mathrm{c}}(L), \leq, \triangleleft_{\mathrm{m}}\right)$.

Proof. Let $f(l):=\left\{j \in \mathcal{J}_{\mathrm{c}}(L) \mid j \leq l\right\}$. Clearly $f(l)$ is a downset, let us verify that it is closed as well: if $j \triangleleft_{\mathrm{m}} C \subseteq f(l)$, then $C \ll l$ and $j \leq \bigvee C \leq l$, so $j \in f(l)$.

Observe now that $f$ is order-preserving; to see that $f$ is an order isomorphism we argue that $\bigvee f(l)=l$ and $f(\bigvee X)=X$, when $X$ is closed subset of $\mathcal{J}_{\mathrm{c}}(L)$.

If $j \leq \bigvee f(l)$, then $j \triangleleft_{\mathrm{m}} C \ll f(l)$; since $f(l)$ is a downset, $C \subseteq f(l)$ follows and therefore $j \in f(l)$, since $f(l)$ is closed; that is, we have $j \leq l$. By spatiality, we have therefore that $\bigvee f(l) \leq l$; equality follows since clearly $l \leq \bigvee f(l)$. For the second relation, if $j \in X$, then $j \leq \bigvee X$ and $j \in f(\bigvee X)$, so $X \subseteq f(\bigvee X)$. Conversely, if $j \in f(\bigvee X)$, then $j \leq \bigvee X$ and $j \triangleleft_{\mathrm{m}} C \ll X$. Since $X$ is a downset, then $C \subseteq X$ and since $X$ is closed, then $j \in X$. Thus $f(\bigvee X) \subseteq X$ and equality holds.

It was shown in 22 how to extend this representation theorem to a duality between the category of finite lattices and the category of OD-graphs. We develop next some observations about pluperfect lattices, that generalize well known facts on finite lattices. We shall need these observations mainly in the course of Section 7 
For a lattice $L$, a principal ideal of $L$ is a subset of the form $\downarrow l:=\{x \in L \mid x \leq l\}$.

Lemma 3. If $L$ is a pluperfect lattice, then every principal ideal $\downarrow l, l \in L$, is also pluperfect. We have $\mathcal{J}_{\mathrm{c}}(\downarrow l)=\mathcal{J}_{\mathrm{c}}(L) \cap \downarrow l$ and, for $\{j\} \cup C \subseteq \mathcal{J}_{\mathrm{c}}(\downarrow l)$, the relation $j \triangleleft_{\mathrm{m}} C$ holds in $\downarrow l$ if and only if it holds in $L$.

Proof. Each element of $\mathcal{J}_{c}(L) \cap \downarrow l$ is completely join-irreducible in $\downarrow l$. If $x \leq l$, then $x=\bigvee J$ with $J \subseteq \mathcal{J}_{c}(L)$ and clearly $J \subseteq \downarrow l$. Therefore $\downarrow l$ is spatial with $\mathcal{J}_{\mathrm{c}}(\downarrow l)=\mathcal{J}_{\mathrm{c}}(L) \cap \downarrow l$.

Suppose now that $\{j\} \cup X \subseteq \downarrow l$ and $j \leq \bigvee X$. If the relations $j \triangleleft_{\mathrm{m}} C$ and $C \ll X$ hold in $L$, then $C \subseteq \downarrow l$, so they hold in $\downarrow l$ as well. In particular, this shows that $\downarrow l$ is pluperfect.

Let $L$ be a pluperfect lattice. A subset $A \subseteq \mathcal{J}_{\mathrm{c}}(L)$ is $D$-closed if $j \in A$ and $j \triangleleft_{\mathrm{m}} C$ implies $C \subseteq A$. Given a $D$-closed subset $A \subseteq \mathcal{J}_{c}(L)$, let $L_{A}$ be the closure of $A$ under possibly infinite joins so, in particular, $L_{A}$ is a sub-join-semilattice of $L$. As $L_{A}$ has infinite joins, it has also infinite meets. Let us define then $\pi_{A}: L \longrightarrow L_{A}$ by $\pi_{A}(l):=\bigvee\left\{x \in L_{A} \mid x \leq l\right\}$. The following Lemma generalizes to pluperfect lattices well known facts about finite lattices, see e.g. [8, Lemma 2.33].

Lemma 4. $\pi_{A}: L \longrightarrow L_{A}$ is a surjective lattice homomorphism. Moreover, $L_{A}$ is a pluperfect lattice whose OD-graph is the restriction to $A$ of the OD-graph of $L$.

Proof. $L_{A}$ is subset of $L$ closed under arbitrary joins and therefore $\pi_{A}: L \longrightarrow L_{A}$, defined by $\pi_{A}(l):=\bigvee\left\{x \in L_{A} \mid x \leq l\right\}$, is a surjective map which preserves arbitrary meets (since meets are computed in $L_{A}$ via this map, e.g. $x \wedge_{L_{A}} y=$ $\left.\pi_{A}\left(x \wedge_{L} y\right)\right)$.

Let us show that $\pi_{A}$ preserves arbitrary joins as well. To this end, observe first that $\pi_{A}(l)=\bigvee\{j \in A \mid j \leq l\}$. Since $\pi_{A}$ is order-preserving, we only need to show that $\pi_{A}(\bigvee X) \leq \bigvee \pi_{A}(X)$. Let therefore $j \in A$ with $j \leq \bigvee X$, so $j \triangleleft_{\mathrm{m}} C$ with $C \ll$ $X$. Since $j \in A$ and $A$ is $D$-closed, we have $C \subseteq A$, whence $C=\pi_{A}(C) \ll \pi_{A}(X)$. It follows that $j \leq \bigvee C \leq \bigvee \pi_{A}(X)$.

The set $A \subseteq \mathcal{J}_{\mathrm{c}}(L)$ generates $L_{A}$ under arbitrary joins and, moreover, each element of $A$ is completely join-irreducible in $L_{A}$, since $L_{A}$ is a sub-join-semilattice of $L$; thus $L_{A}$ is spatial and $\mathcal{J}_{\mathrm{c}}\left(L_{A}\right)=A$. It is easily verified that, for each $j \in A$, each minimal join-cover of $j L$ is also a minimal join-cover of $j$ in $L_{A}$.

Lemma 5. If $\left\{A_{i} \mid i \in I\right\}$ is a collection of D-closed subsets such that $\bigcup A_{i}=$ $\mathcal{J}_{\mathrm{c}}(L)$, then $\left\langle\pi_{A_{i}} \mid i \in I\right\rangle: L \longrightarrow \prod L_{A_{i}}$ is a lattice embedding, that is, a subdirect decomposition of $L$.

Proof. If $l \not \leq l^{\prime}$, then, by spatiality, there is $j \in \mathcal{J}_{\mathrm{c}}(L)$ such that $j \leq l$ but $j \not \leq l^{\prime}$. Let $i \in I$ such that $j \in A_{i}$ : then $j \leq \pi_{A_{i}}(l)$ but $j \not \leq \pi_{A_{i}}\left(l^{\prime}\right)$. It follows that $\left\langle\pi_{A_{i}} \mid i \in I\right\rangle$ is an injective map.

\section{The Relational lattices $\mathrm{R}(D, A)$}

Throughout this paper we shall use the notation $Y^{X}$ for the set of functions of domain $Y$ and codomain $X$, for $X$ and $Y$ any two sets.

Let $A$ be a collection of attributes (or column names) and let $D$ be a set of cell values. A relation on $A$ and $D$ is a pair $(\alpha, T)$ where $\alpha \subseteq A$ and $T \subseteq D^{\alpha}$. We shall use $\mathrm{R}(D, A)$ to denote the set of relations on $A$ and $D$. Informally, a relation 
$(\alpha, T)$ represents a table of a relational database, with $\alpha$ being the header, i.e. the collection of names of columns, while $T$ is the collection of rows.

Before we define the natural join, the inner union operations, and the order on $\mathrm{R}(D, A)$, let us recall some key operations. If $\alpha \subseteq \beta \subseteq A$ and $f \in D^{\beta}$, then we shall use $f_{\uparrow_{\alpha}} \in D^{\alpha}$ for the restriction of $f$ to $\alpha$; if $T \subseteq D^{\beta}$, then $T \|_{\alpha}$ shall denote projection to $\alpha$, that is, the direct image of $T$ along restriction, $T \|_{\alpha}:=\left\{f_{\uparrow_{\alpha}} \mid f \in T\right\}$; if $T \subseteq D^{\alpha}$, then $i_{\beta}(T)$ shall denote cylindrification to $\beta$, that is, the inverse image of restriction, $i_{\beta}(T):=\left\{f \in D^{\beta} \mid f_{\lceil\alpha} \in T\right\}$. Recall that $i_{\beta}$ is right adjoint to $\|_{\alpha}$. With this in mind, the natural join $\bowtie$ and the inner union $\oplus$ of relations are respectively described by the following formulas:

$$
\begin{aligned}
\left(\alpha_{1}, T_{1}\right) \bowtie\left(\alpha_{2}, T_{2}\right) & :=\left(\alpha_{1} \cup \alpha_{2}, T\right) \\
\text { where } T & =\left\{f \mid f_{\left\lceil\alpha_{i}\right.} \in T_{i}, i=1,2\right\} \\
& =i_{\alpha_{1} \cup \alpha_{2}}\left(T_{1}\right) \cap i_{\alpha_{1} \cup \alpha_{2}}\left(T_{2}\right), \\
\left(\alpha_{1}, T_{1}\right) \oplus\left(\alpha_{2}, T_{2}\right) & :=\left(\alpha_{1} \cap \alpha_{2}, T\right) \\
\text { where } T & =\left\{f \mid \exists i \in\{1,2\}, \exists g \in T_{i} \text { s.t. } g_{\uparrow_{\alpha_{1} \cap \alpha_{2}}}=f\right\} \\
& =T_{1}\left\|\alpha_{1} \cap \alpha_{2} \cup T_{2}\right\| \alpha_{1} \cap \alpha_{2} .
\end{aligned}
$$

The set $\mathrm{R}(D, A)$ is then ordered as follows:

$$
\left(\alpha_{1}, T_{1}\right) \leq\left(\alpha_{2}, T_{2}\right) \quad \text { iff } \quad \alpha_{2} \subseteq \alpha_{1} \text { and } T_{1} \| \alpha_{2} \subseteq T_{2} .
$$

Proposition 6 (Tropashko [26]). The poset $(\mathrm{R}(D, A), \leq)$ is a lattice, with $\bowtie$ as the meet operation and $\oplus$ as the join operation.

We shall therefore use $\mathrm{R}(D, A)$ to denote such a lattice and call it a relational lattice. Let us remark however that in [16] such a lattice is called full relational lattice and that the wording "class of relational lattices" is used there for the class of lattices that have an embedding into some lattice of the form $\mathrm{R}(D, A)$. As our concerns are lattice theoretical, we shall avoid to use the symbols $\bowtie$ and $\oplus$, and prefer instead the usual meet and join symbols $\wedge$ and $\vee$.

A convenient way of describing these lattices was introduced in [16, Lemma 2.1]. The authors argued that the relational lattices $\mathrm{R}(D, A)$ are isomorphic to the lattices of closed subsets of $A \cup D^{A}$, where $Z \subseteq A \cup D^{A}$ is said to be closed if it is a fixed-point of the closure operator $\overline{(-)}$ defined as

$$
\bar{Z}:=Z \cup\left\{f \in D^{A} \mid A \backslash Z \subseteq E q(f, g) \text {, for some } g \in Z\right\},
$$

where in the formula above $E q(f, g)$ is the equalizer of $f$ and $g$. Letting

$$
\delta(f, g):=\{x \in A \mid f(x) \neq g(x)\},
$$

the above definition of the closure operator is obviously equivalent to the following one:

$$
\bar{Z}:=\alpha \cup\left\{f \in D^{A} \mid \delta(f, g) \subseteq \alpha, \text { for some } g \in\left(Z \cap D^{A}\right)\right\}, \text { with } \alpha=Z \cap A .
$$

From now on, we rely on this representation of relational lattices. Relational lattices are atomistic pluperfect lattices. The completely join-irreducible elements of $\mathrm{R}(D, A)$ are the singletons $\{a\}$ and $\{f\}$, for $a \in A$ and $f \in D^{A}$, see [16]. By an abuse of notation we shall write $x$ for the singleton $\{x\}$, for $x \in A \cup D^{A}$. Under this 
convention, we have therefore $\mathcal{J}_{\mathrm{c}}(\mathrm{R}(D, A))=A \cup D^{A}$. Every $a \in A$ is join-prime, while the minimal join-covers are of the form

$$
f \triangleleft_{\mathrm{m}} \delta(f, g) \cup\{g\}
$$

for each $f, g \in D^{A}$, see 23 . The only non-trivial result from 23 that we use later (for Theorem 43 and Lemma 50) is the following:

Lemma 7. Let $L$ be a finite atomistic lattice in the variety generated by the class of relational lattices. If $\{j\} \cup X \subseteq \mathcal{J}_{\mathrm{c}}(L), j \leq \bigvee X$, and all the elements of $X$ are join-prime, then $j$ is join-prime.

The Lemma - which is an immediate consequence of Theorem 7 in 23] - asserts that a join-cover of an element $j \in \mathcal{J}_{c}(L)$ which is not join-prime cannot be made of join-prime elements only.

\section{Overview And statement of the Results}

For an arbitrary frame $\mathfrak{F}$, we construct in Section 5 a lattice $L(\mathfrak{F})$; if $\mathfrak{F}$ is rooted and full, then $L(\mathfrak{F})$ is a subdirectly irreducible lattice, see Proposition 23. The key Theorem leading to the undecidability results is the following one.

Theorem 8. Let $A$ be a finite set and let $\mathfrak{F}$ be an $\mathrm{S} 4$ finite rooted full $A$-frame. There is a surjective p-morphism from a universal $\mathrm{S} 5^{A}$-product frame $\mathfrak{U}$ to $\mathfrak{F}$ if and only if $\mathrm{L}(\mathfrak{F})$ embeds into some relational lattice $\mathrm{R}(D, B)$.

outline. The construction $L$ defined in Section 5 extends to a contravariant functor, so if $\mathfrak{U}$ is a universal $\mathrm{S} 5^{A}$-product frame and $\psi: \mathfrak{U} \longrightarrow \mathfrak{F}$ is a surjective $p$-morphism, then we have an embedding $\mathrm{L}(\psi)$ of $L(\mathfrak{F})$ into $\mathrm{L}(\mathfrak{U})$. We can assume that all the components of $\mathfrak{U}$ are equal, i.e. that the underlying set of $\mathfrak{U}$ is of the form $\prod_{a \in A} X$; if this is the case, then $\mathrm{L}(\mathfrak{U})$ is isomorphic to the relational lattice $\mathrm{R}(X, A)$.

The converse direction, developed from Section 6 up to Section 8 is subtler. Considering that $\mathrm{L}(\mathfrak{F})$ is subdirectly-irreducible, we argue in Section 7 that if $\psi$ : $\mathrm{L}(\mathfrak{F}) \longrightarrow \mathrm{R}(D, B)$ is a lattice embedding, then we can suppose it preserves bounds; in this case $\psi$ has a surjective left adjoint $\mu: \mathrm{R}(D, B) \longrightarrow \mathrm{L}(\mathfrak{F})$. Let us notice that there is no general reason for $\psi$ to be the image by $L$ of a $p$-morphism. Said otherwise, the functor $L$ is not full and, in particular, the image of an atom by $\mu$ might not be an atom. The following considerations, mostly developed in Section 8 make it possible to extract a $p$-morphism from the left adjoint $\mu$. Since both $\mathrm{L}(\mathfrak{F})$ and $\mathrm{R}(D, B)$ are generated (under possibly infinite joins) by their atoms, each atom $x \in \mathrm{L}(\mathfrak{F})$ has a preimage $y \in \mathrm{R}(D, B)$ which is an atom. The set $F_{0}$ of non-joinprime atoms of $\mathrm{R}(D, B)$ such that $\mu(f)$ is a non-join-prime atom of $\mathrm{L}(\mathfrak{F})$ is endowed with a $P(A)$-valued distance $\delta$. The pair $\left(F_{0}, \delta\right)$ is shown to be a pairwise complete ultrametric space over $P(A)$. Section 6 recalls and develops some observations on ultrametric spaces valued on powerset algebras. The key ones are Theorems 27 and 39, stating that - when $A$ is finite - pairwise complete ultrametric spaces over $P(A)$ and universal $\mathrm{S} 5^{A}$-product frames are essentially the same objects. The restriction of $\mu$ to $F_{0}$ yields then a surjective $p$-morphism from $F_{0}$, considered as a universal $\mathrm{S} 5{ }^{A}$-product frame, to $\mathfrak{F}$.

The following problem was shown to be undecidable in [12] given a finite 3frame $\mathfrak{F}$, does there exists a surjective $p$-morphism from a universal $\mathrm{S} 5^{3}$-product frame $\mathfrak{U}$ to $\mathfrak{F}$ ? In the introduction we referred to this problem as the coverability 
problem of a 3 -frame by a universal $\mathrm{S} 5^{3}$-product frame. The problem was shown to be undecidable by means of a reduction from the representability problem of finite simple relation algebras, shown to be undecidable in [11. We need to strengthen the undecidability result of [12] with some additional observations - rootedness and fullness - as stated in the following Proposition.

Proposition 9. It is undecidable whether, given a finite set $A$ with $\operatorname{card} A \geq 3$ and an S4 finite rooted full $A$-frame $\mathfrak{F}$, there is a surjective p-morphism from a universal $\mathrm{S} 5^{A}$-product $\mathfrak{U}$ to $\mathfrak{F}$.

Proof. Throughout this proof we assume a minimum knowledge of the theory of relation algebras, see e.g. [18.

The Proposition actually holds if we restrict to the case when $\operatorname{card} A=3$. Given a finite simple relation algebra $\mathfrak{A}$, the authors of [12] construct a 3 -multimodal frame $\mathfrak{F}_{\mathfrak{A}, 3}$ such that $\mathfrak{A}$ is representable if and only if $\mathfrak{F}_{\mathfrak{A}, 3}$ is a $p$-morphic image of some universal $\mathrm{S}^{3}$-product frame. The frame $\mathfrak{F}_{\mathfrak{A}, 3}$ is $\mathrm{S} 4$ and rooted [12, Claim 8]. We claim that $\mathfrak{F}_{\mathfrak{A}, 3}$ is also full, unless $\mathfrak{A}$ is the two elements Boolean algebra. To prove this claim, let us recall first that an element of $\mathfrak{F}_{\mathfrak{A}, 3}$ is a triple $\left(t_{0}, t_{1}, t_{2}\right)$ of atoms of $\mathfrak{A}$ such that $t_{2}^{\smile} \leq t_{0} ; t_{1}$; moreover, if $t, t^{\prime}$ are two such triples and $i \in\{0,1,2\}$, then $t R_{i} t^{\prime}$ if and only if $t$ and $t^{\prime}$ coincide in the $i$-th coordinate. If $a$ is an atom of $\mathfrak{A}$, then $a \leq e_{l} ; a$ and $a \leq a ; e_{r}$ for two atoms $e_{l}, e_{r}$ below the multiplicative unit of $\mathfrak{A}$. Therefore, the triples $t:=\left(e_{l}, a, a^{\smile}\right)$ and $t^{\prime}=\left(a, e_{r}, a^{\smile}\right)$ are elements of $\mathfrak{F}_{\mathfrak{A}, 3}$ and $t R_{2} t^{\prime}$. If, for each atom $a$, these triples are equal, then every atom of $\mathfrak{A}$ is below the multiplicative unit, which therefore concides with the top element $T$; since $\mathfrak{A}$ is simple, then relation $\top=\top ; x ; \top$ holds for each $x \neq \perp$. It follows that $x=\top ; x ; \top=\top$, for each $x \neq \perp$, so $\mathfrak{A}$ is the two elements Boolean algebra. Thus, if $\mathfrak{A}$ has more than two elements, then $t \neq t^{\prime}$ and $t R_{2} t^{\prime}$ for some $t, t^{\prime} \in \mathfrak{F}_{\mathfrak{A}, 3}$. Using the cycle law of relation algebras, one also gets pairs of distinct elements of $\mathfrak{F}_{\mathfrak{A}, 3}$, call them $u, u^{\prime}$ and $w, w^{\prime}$, such that $u R_{0} u^{\prime}$ and $w R_{1} w^{\prime}$.

Therefore, if we could decide whether there is a $p$-morphism from some universal $\mathrm{S} 5^{3}$-frame to a given $\mathrm{S} 4$ finite rooted full frame $\mathfrak{F}$, then we could also decide whether a finite simple relation algebra $\mathfrak{A}$ is representable, by answering positively if $\mathfrak{A}$ has exactly two elements and, otherwise, by answering the existence problem of a $p$ morphism to $\mathfrak{F}_{\mathfrak{A}, 3}$.

Combining Theorem 8 with Proposition 9, we derive the following undecidability result.

Theorem 10. It is not decidable whether a finite subdirectly irreducible atomistic lattice embeds into a relational lattice.

Let us remark that Theorem 10 partly answers Problem 7.1 in [16].

In [16] the authors proved that the quasiequational theory of relational lattices (i.e. the set of all definite Horn sentences valid in relational lattices) in the signature $(\wedge, \vee, H)$ is undecidable. Here $H$ is the header constant, which is interpreted in a relational lattice $\mathrm{R}(D, A)$ as the closed subset $A$ of $A \cup D^{A}$. Problem 4.10 in 16] asks whether the quasiequational theory of relational lattices in the restricted signature $(\wedge, \vee)$ of pure lattice theory is undecidable as well. We positively answer this question.

Theorem 11. The quasiequational theory of relational lattices in the pure lattice signature is undecidable. 
It is a general fact that if the embeddability problem of finite subdirectlyirreducible algebras in a class $\mathcal{K}$ is undecidable, then the quasiequational theory of $\mathcal{K}$ is undecidable as well. We thank one of the authors of [13] for pointing out to us how this can be derived from Evans' work 7] as well as the connection to their work. We add here the proof of this fact, since we shall need it later in the proof of Theorem 14.

Proof of Theorem 11. Given a finite subdirectly-irreducible algebra $A$ with least non trivial congruence $\theta(\hat{a}, \bar{a})$, we construct a quasiequation $\phi_{A}$ with the following property: for any other algebra (in the same signature) $K, K \forall \phi_{A}$ if and only if $A$ has an embedding into $K$.

The construction is as follows. Let $X_{A}=\left\{x_{a} \mid a \in A\right\}$ be a set of variables in bijection with the elements of $A$. For each function symbol $f$ in the signature $\Omega$, let $T_{A, f}$ be its table, that is the formula

$$
T_{A, f}=\bigwedge_{\left(a_{1}, \ldots, a_{a r(f)}\right) \in A^{a r(f)}} f\left(x_{a_{1}}, \ldots, x_{a r(f)}\right)=x_{f\left(a_{1}, \ldots, a_{a r(f)}\right)} .
$$

We let $\phi_{A}$ be the universal closure of $\bigwedge_{f \in \Omega} T_{A, f} \Rightarrow x_{\hat{a}}=x_{\bar{a}}$. We prove next that an algebra $K$ sastifies $\phi_{A}$ if and only if there is no embedding of $A$ into $K$.

If $K \models \phi_{A}$ and $\psi: A \longrightarrow K$, then $v\left(x_{a}\right)=\psi(a)$ is a valuation such that $K, v \models \bigwedge_{f \in \Omega} T_{A, f}$, so $\psi(\hat{a})=v\left(x_{\hat{a}}\right)=v\left(x_{\bar{a}}\right)=\psi(\bar{a})$ and $\psi$ is not injective.

Conversely, suppose $K \forall \phi_{A}$ and let $v$ be a valuation such that $K, v \models \bigwedge_{f \in \Omega} T_{A, f}$ and $K, v \not \forall x_{\hat{a}}=x_{\bar{a}}$. Define $\psi: A \longrightarrow K$ as $\psi(a)=v\left(x_{a}\right)$, then $\psi$ is a morphism, since $K, v \models T_{A, f}$ for each $f \in \Omega$. Let $\operatorname{Ker}_{\psi}=\left\{\left(a, a^{\prime}\right) \mid \psi(a)=\psi\left(a^{\prime}\right)\right\}$ so, supposing that $\psi$ is not injective, $\mathrm{Ker}_{\psi}$ is a non-trivial congruence. Then $(\hat{a}, \bar{a}) \in \theta(\hat{a}, \bar{a}) \subseteq \operatorname{Ker}_{\psi}$, so $v\left(x_{\hat{a}}\right)=\psi(\hat{a})=\psi(\bar{a})=v\left(x_{\bar{a}}\right)$, a contradiction. We have therefore $\operatorname{Ker}_{\psi}=\{(a, a) \mid a \in A\}$, which shows that $\psi$ is injective.

Let now $\mathcal{K}$ be a class of algebras in the same signature. We have then

$$
\mathcal{K} \forall \phi_{A} \text { iff } K \forall \phi_{A} \text { for some } K \in \mathcal{K}
$$

iff there is an embedding of $A$ into $K$, for some $K \in \mathcal{K}$.

Thus, if the embeddability problem of finite subdirectly-irreducible algebras into some algebra in $\mathcal{K}$ is undecidable, then the quasiequational theory of $\mathcal{K}$ is undecidable as well.

Following [12, let us add some further observations on the quasiequational theory of relational lattices.

Lemma 12. The class of lattices that have an embedding into a relational lattice is closed under ultraproducts.

Proof. Let us say that a sublattice $L$ of a lattice $\mathrm{R}(D, A)$ is $H$-closed if the subset $A$ belongs to $L$. Let $\mathcal{R}$ denote the closure under isomorphisms of the class of $H$-closed sublattices of some $\mathrm{R}(D, A)$. It is proved in [16. Corollary 4.2] that $\mathcal{R}$ is closed under ultraproducts. It immediately follows from this result that the class of lattices that have an embedding into some relational lattice is closed under ultraproducts, as follows. Let $\left\{L_{i} \longrightarrow \mathrm{R}\left(D_{i}, A_{i}\right) \mid i \in I\right\}$ be a family of lattice embeddings and let $\mathcal{F}$ be an ultrafilter over $I$. The ultraproduct constructions on $\left\{L_{i} \mid i \in I\right\}$ and $\left\{\mathrm{R}\left(D_{i}, A_{i}\right) \mid i \in I\right\}$ yield a lattice embedding $\prod_{\mathcal{F}} L_{i} \longrightarrow \prod_{\mathcal{F}} \mathrm{R}\left(D_{i}, A_{i}\right)$. Clearly, each $\mathrm{R}\left(D_{i}, A_{i}\right)$ belongs to $\mathcal{R}$, whence the ultraproduct $\prod_{\mathcal{F}} \mathrm{R}\left(D_{i}, A_{i}\right)$ belongs to $\mathcal{R}$ as well: thus $\prod_{\mathcal{F}} \mathrm{R}\left(D_{i}, A_{i}\right)$ embeds into some $\mathrm{R}(D, A)$, and so does $\prod_{\mathcal{F}} L_{i}$. 
Theorem 13. The quasiequational theory of relational lattices is not finitely axiomatizable.

Proof. A known result in universal algebra - see e.g. [3, Theorem 2.25] - states that a subdirectly-irreducible algebra satisfies all the quasiequations satisfied by a class of algebras if and only if it embeds in an ultraproduct of algebras in this class. Lemma 12 implies that the class of lattices that have an embedding into an ultraproduct of relational lattices and the class of lattices that have an embedding into some relational lattices are the same. Therefore a subdirectly-irreducible lattice $L$ embeds in a relational lattice if and only if it satisfies all the quasiequations satisfied by the relational lattices. If this collection of quasiequations was a logical consequence of a finite set of quasiequations, then we could decide whether a finite subdirectly-irreducible $L$ satisfies all these quasiequations, by verifying whether $L$ satisfies the finite set of quasiequations. In this way, we could also decide whether such an $L$ embeds into some relational lattice.

Finally, the following Theorem, showing that the quasiequational theory of the finite relational lattices is stronger than the quasiequational theory of all the relational lattices, partly answers Problem 3.6 in [16.

Theorem 14. There is a quasiequation which holds in all the finite relational lattices which, however, fails into an infinite relational lattice.

Proof. In the first appendix of [12] an S4 finite rooted full 3 -frame $\mathfrak{F}$ is constructed that has no surjective $p$-morphism from a finite universal $\mathrm{S} 5^{3}$-product frame, but has such a $p$-morphism from an infinite one.

Since $L(\mathfrak{F})$ is finite whenever $\mathfrak{F}$ is finite, we obtain by using Theorem 8 a subdirectlyirreducible finite lattice $L$ which embeds into an infinite relational lattice, but has no embedding into a finite one.

Let $\phi_{L}$ be the quasiequation as in the proof of Theorem 11] We have therefore that, for any lattice $K, K \models \phi_{L}$ if and only if $L$ does not embed into $K$.

Correspondingly, any finite relational lattice satisfies $\phi_{L}$ and, on the other hand, $K \not \phi_{L}$ if $K$ is the infinite lattice into which $L$ embeds.

\section{The lattice of a multimodal frame}

We assume throughout this Section that $A$ is a finite set of actions. Given an $A$-frame $\mathfrak{F}=\left\langle X_{\mathfrak{F}},\left\{R_{a} \mid a \in A\right\}\right\rangle$, we construct a lattice as follows. For $\alpha \subseteq A$, we say that $Y \subseteq X_{\mathfrak{F}}$ is $\alpha$-closed if $x \in Y$, whenever there is a $\alpha$-path from $x$ to some $y \in Y$. We say that a subset $Z \subseteq A \cup X_{\mathfrak{F}}$ is closed if $Z \cap X_{\mathfrak{F}}$ is $Z \cap A$-closed.

Lemma 15. The collection of closed subsets of $A \cup X$ is a Moore family.

The Lemma, whose proof is straightforward, allows us to define the lattice of an $A$-frame.

Definition 16. The lattice $\mathrm{L}(\mathfrak{F})$ is the lattice of closed subsets of $A \cup X_{\mathfrak{F}}$.

The lattice operations on $L(\mathfrak{F})$ are defined as in the display (1). In order to master the formula for the join, we need a more explicit description of the closure operator associated to this Moore family. If $\alpha \subseteq A$ and $Y \subseteq X_{\mathfrak{F}}$, define

$$
\bar{Y}^{\alpha}:=\left\{x \in X_{\mathfrak{F}} \mid \exists y \in Y, x \stackrel{\alpha}{\rightarrow} y\right\} .
$$


Lemma 17. For $Z \subseteq A \cup X_{\mathfrak{F}}$, we have

$$
\bar{Z}=\alpha \cup{\overline{Z \cap X_{\mathfrak{F}}}}^{\alpha}, \quad \text { where } \alpha=Z \cap A .
$$

In particular, for $x \in X_{\mathfrak{F}}, x \in \bar{Z}$ if and only if there exists $y \in Z \cap X_{\mathfrak{F}}$ and an $\alpha$-path from $x$ to $y$, with $\alpha=Z \cap A$.

The above formula (2) allows to make $\mathrm{L}(-)$ into a contravariant functor from the category of frames to the category of lattices. Namely, for a $p$-morphism $\psi$ : $\mathfrak{F}_{0} \longrightarrow \mathfrak{F}_{1}$ and any $Z \subseteq A \cup X_{\mathfrak{F}_{1}}$, define

$$
\mathrm{L}(\psi)(Z):=(Z \cap A) \cup \psi^{-1}\left(Z \cap X_{\mathfrak{F}_{1}}\right) .
$$

Let $\psi^{A}: A \cup X_{\mathfrak{F}_{0}} \longrightarrow A \cup X_{\mathfrak{F}_{1}}$ be the function such that $\psi^{A}(a)=a$, for each $a \in A$, and $\psi^{A}(x)=\psi(x)$, for each $x \in X_{\mathfrak{F}_{0}}$. Notice that $\mathrm{L}(\psi)$ is the inverse image of $\psi^{A}$, so in particular $\mathrm{L}(\psi)$ commutes with intersections and unions.

Proposition 18. $\mathrm{L}(\psi)$ sends closed subsets of $A \cup X_{\mathfrak{F}_{1}}$ to closed subsets of $A \cup X_{\mathfrak{F}_{0}}$. Its restriction to $\mathrm{L}\left(\mathfrak{F}_{1}\right)$ yields a bound-preserving lattice morphism $L(\psi): \mathrm{L}\left(\mathfrak{F}_{1}\right) \longrightarrow$ $\mathrm{L}\left(\mathfrak{F}_{0}\right)$.

Proof. The key observation is that, for each $\alpha \subseteq A$ and each $Y \subseteq X_{\mathfrak{F}_{1}}$, we have $\psi^{-1}\left(\bar{Y}^{\alpha}\right)={\overline{\psi^{-1}(Y)}}^{\alpha}$ :

$$
\begin{aligned}
\psi^{-1}\left(\bar{Y}^{\alpha}\right) & =\psi^{-1}\left(\left\{x \in X_{\mathfrak{F}_{1}} \mid \exists y \in Y, x \stackrel{\alpha}{\rightarrow} y\right\}\right) \\
& =\left\{x \in X_{\mathfrak{F}_{0}} \mid \exists y \in Y, \psi(x) \stackrel{\alpha}{\rightarrow} y\right\} \\
& =\left\{x \in X_{\mathfrak{F}_{0}} \mid \exists z \in \psi^{-1}(Y), x \stackrel{\alpha}{\rightarrow} z\right\} \quad \text { since } \psi \text { is a } p \text {-morphism, } \\
& =\bar{\psi}^{-1}(Y)
\end{aligned}
$$

This implies that, for $Z \subseteq A \cup X_{\mathfrak{F}_{1}}$, we have

$$
\mathrm{L}(\psi)(\bar{Z})=\overline{\mathrm{L}(\psi)(Z)} .
$$

In particular, if $Z \subseteq A \cup X_{\mathfrak{F}_{1}}$ is closed, then

$$
\mathrm{L}(\psi)(Z)=\mathrm{L}(\psi)(\bar{Z})=\overline{\mathrm{L}(\psi)(Z)}
$$

so $\mathrm{L}(\psi)$ sends closed subsets to closed subsets. $\mathrm{L}(\psi)$ preserves all meets, since it commutes with intersections. Moreover

$$
\mathrm{L}(\psi)\left(\bigvee_{i \in I} Z_{i}\right)=\mathrm{L}(\psi)\left(\overline{\bigcup_{i \in I} Z_{i}}\right)=\overline{\bigcup_{i \in I} \mathrm{~L}(\psi)\left(Z_{i}\right)}=\bigvee_{i \in I} \mathrm{~L}(\psi)\left(Z_{i}\right),
$$

so $\mathrm{L}(\psi)$ is a lattice morphism.

As $\mathrm{L}(\psi)$ is the restriction of the inverse image of $\psi^{A}$ defined above, it immediately follows that $\mathrm{L}$ is a contravariant functor from the category of $A$-frames to the category of lattices.

Lemma 19. If $\psi: \mathfrak{F}_{0} \longrightarrow \mathfrak{F}_{1}$ is surjective, then $\mathrm{L}(\psi)$ is injective.

Proof. If $\psi$ is surjective, then $\psi^{A}$ is also surjective. $\operatorname{As~} \mathrm{L}(\psi)$ is the inverse image of $\psi^{A}$, then $\mathrm{L}(\psi)$ is injective.

We are ready to state the main result of this Section.

Theorem 20. If there exists a surjective p-morphism from a universal $\mathrm{S} 5^{A}$-product frame $\mathfrak{U}$ to an $A$-frame $\mathfrak{F}$, then $\mathrm{L}(\mathfrak{F})$ embeds into a relational lattice. 
Proof. We say that $\mathfrak{U}$ is uniform on $X$ if all the components of $\mathfrak{U}$ are equal to $X$. Spelled out, this means that $X_{\mathfrak{U}}=\prod_{a \in A} X$. Let $\psi: \mathfrak{U} \longrightarrow \mathfrak{F}$ be a $p$-morphism as in the statement of the Theorem. W.l.o.g. we can assume that $\mathfrak{U}$ is uniform on some set $X$. If this is not the case, then we choose $a_{0} \in A$ such that $X_{a_{0}}$ has maximum cardinality and surjective mappings $p_{a}: X_{a_{0}} \longrightarrow X_{a}$, for each $a \in$ $A$. The product frame $\mathfrak{U}^{\prime}$ on $\prod_{a \in A} X_{a_{0}}$ is uniform and $\prod_{a \in A} p_{a}: \mathfrak{U}^{\prime} \longrightarrow \mathfrak{U}$ is a surjective $p$-morphism. By pre-composing $\psi$ with this $p$-morphism, we obtain a surjective $p$-morphism from the uniform $\mathfrak{U}^{\prime}$ to $\mathfrak{F}$. Now, if $\mathfrak{U}$ is uniform on $X$, then $\mathrm{L}(\mathfrak{U})$ is equal to the relational lattice $\mathrm{R}(X, A)$. Then, by functoriality of $\mathrm{L}$, we have a lattice morphism

$$
\mathrm{L}(\psi): \mathrm{L}(\mathfrak{F}) \longrightarrow \mathrm{L}(\mathfrak{U})=\mathrm{R}(X, A) .
$$

By Lemma $19 \mathrm{~L}(\psi)$ is an embedding.

Some properties of the lattices $L(\mathfrak{F})$.

Proposition 21. The completely join-irreducible elements of $\mathrm{L}(\mathfrak{F})$ are the singletons, so $\mathrm{L}(\mathfrak{F})$ is an atomistic lattice.

Proof. Each singleton set is closed. It immediately follows that the join-irreducible elements of $\mathrm{L}(\mathfrak{F})$ are the singletons and clearly they are atoms.

Identifying the singletons of $P\left(A \cup X_{\mathfrak{F}}\right)$ with their elements, we can write

$$
\mathcal{J}_{c}(\mathrm{~L}(\mathfrak{F}))=A \cup X_{\mathfrak{F}} .
$$

To state the next Proposition, let us say that an $\alpha$-path from $x$ to $y$ is minimal if there is no $\beta$-path from $x$ to $y$, for each proper subset $\beta$ of $\alpha$.

Proposition 22. $\mathrm{L}(\mathfrak{F})$ is a pluperfect lattice. Each element of $A$ is join-prime, while the minimal join-covers of $x \in X_{\mathfrak{F}}$ are of the form $x \triangleleft_{\mathrm{m}} \alpha \cup\{y\}$, for a minimal $\alpha$-path from $x$ to $y$.

Proof. We observe that, for $Z \subseteq A \cup X_{\mathfrak{F}}$, the relation

$$
x \in \bigvee Z=\bar{Z}
$$

holds if and only if either (i) $x \in A \cap Z$, or (ii) $x \in X_{\mathfrak{F}}$ and $x \stackrel{\alpha}{\rightarrow} y$ for some $y \in X_{\mathfrak{F}} \cap Z$ and some $\alpha \subseteq A \cap Z$. Thus, in particular, each element of $A$ is joinprime. If $x \in X_{\mathfrak{F}}, Z \subseteq A \cup X_{\mathfrak{F}}$ and $x \leq \bigvee Z$, then we can find $y \in X_{\mathfrak{F}} \cap Z$ and an $\alpha$-path from $x$ to $y$ with $\alpha \subseteq A \cap Z$. Clearly, we can assume $x \stackrel{\alpha}{\rightarrow} y$ is minimal, so

$$
x \in \overline{\alpha \cup\{y\}}=\bigvee \alpha \vee y,
$$

with $\alpha \cup\{y\} \subseteq Z$. This proves that every cover of $x$ refines to a cover of the form $x \leq \bigvee \alpha \vee y$ with $x \stackrel{\alpha}{\rightarrow} y$ minimal.

Notice that if an $\alpha$-path is minimal, then $\alpha$ is necessarily finite. Therefore $\mathrm{L}(\mathfrak{F})$ is actually a lattice with the $\Sigma$-weak minimal join-cover refinement property as defined in [27, where $\Sigma$ is here the set of completely join-irreducible elements of the lattice.

Before stating the next Proposition, let us recall from [8, Corollary 2.37], see also [22, Section 5.2], that a finite lattice $L$ is subdirectly-irreducible if and only if 
the directed graph $\left(\mathcal{J}_{\mathrm{c}}(L), D\right)$ is rooted. Here $D$ is the join-dependency relation on the join-irreducible elements of $L$, defined as follows:

$$
j D k \text { iff } j \neq k \text { and, for some } p \in L, j \leq p \vee k \text { and } j \neq p \vee k_{*},
$$

where $k_{*}$ denotes the unique lower cover of $k \in \mathcal{J}_{c}(L)$. It can be shown that $j D k$ if and only if $k \neq j$ and $k \in C$ for some subset $C \subseteq \mathcal{J}_{\mathrm{c}}(L)$ such that $j \triangleleft_{\mathrm{m}} C$, see e.g. [8, Lemma 2.31]. If a lattice is atomistic, then $k_{*}=\perp$ for each $k \in \mathcal{J}_{c}(L)$, and therefore $j D k$ if and only if $j \neq k$ and $j \leq p \vee k$ for some $p \in L$ with $j \not \leq p$.

Proposition 23. If a finite A-frame $\mathfrak{F}$ is rooted and full, then $\mathrm{L}(\mathfrak{F})$ is a subdirectlyirreducible lattice.

Proof. We argue that the digraph $\left(\mathcal{J}_{\mathrm{c}}(\mathrm{L}(\mathfrak{F})), D\right)$ is rooted. Observe that $x \in$ $\overline{\{a, y\}}=a \vee y$ whenever $x R_{a} y$. This implies that $x D y$ and $x D a$ when $x, y \in X_{\mathfrak{F}}$, $a \in A, x \neq y$ and $x R_{a} y$. The fact that of $\left(\mathcal{J}_{c}(\mathrm{~L}(\mathfrak{F})), D\right)$ is rooted follows now from $\mathfrak{F}$ being rooted and full.

\section{Some THEORY of GENERALized UltrametriC SPACES}

Generalized ultrametric spaces over a Boolean algebra $P(A)$ turn out to be a useful tool for relational lattices [16, 23] - as well as, we claim here, for universal product frames from multidimensional modal logic [14. The use of metrics is well known in graph theory, where universal product frames are known as Hamming graphs, see e.g. [10. Generalized ultrametric spaces over a Boolean algebra $P(A)$ were introduced in 20 to study equivalence relations. The main results of this Section are Theorem 27 and Proposition 39 which together substantiate the claim that when $A$ is finite, universal $S 5^{A}$-product frames are pairwise complete ultrametric spaces valued in the Boolean algebra $P(A)$. It is this abstract point of view that shall allow us to construct a universal product frame given a lattice embedding $\mathrm{L}(\mathfrak{F}) \longrightarrow \mathrm{R}(D, A)$.

Some of the observations we shall develop are not strictly necessary to prove the undecidability result, which is the main result of this paper; namely, we can always suppose that the set $A$ is finite. Nonetheless we include these observations since they are part of a coherent set of results and, as far as we are aware of, they are original.

Definition 24. An ultrametric space over $P(A)$ (briefly, a space) is a pair $(X, \delta)$, with $\delta: X \times X \longrightarrow P(A)$ such that, for every $f, g, h \in X$,

$$
\delta(f, f) \subseteq \emptyset, \quad \delta(f, g) \subseteq \delta(f, h) \cup \delta(h, g) .
$$

That is, we have defined an ultrametric space over $P(A)$ as a category (with a small set of objects) enriched over $\left(P(A)^{o p}, \emptyset, \cup\right)$, see 15. We shall assume in this paper that such a space $(X, \delta)$ is also reduced and symmetric, that is, that the following two properties hold for every $f, g \in X$ :

$$
\delta(f, g)=\emptyset \text { implies } f=g, \quad \delta(f, g)=\delta(g, f) .
$$

Under these hypothesis, it is easily seen that if $A$ is empty or a singleton, then the categories of spaces over $P(A)$ are trivial. Thus, we shall assume here that $A$ has at least two elements. 
A morphism of spaces $\psi:\left(X, \delta_{X}\right) \longrightarrow\left(Y, \delta_{Y}\right)$ is a function $\psi: X \longrightarrow Y$ such that $\delta_{Y}(\psi(f), \psi(g)) \leq \delta_{X}(f, g)$, for each $f, g \in X$. If $\delta_{Y}(\psi(f), \psi(g))=\delta_{X}(f, g)$, for each $f, g \in X$, then $\psi$ is said to be an isometry. For $(X, \delta)$ a space over $P(A), f \in X$ and $\alpha \subseteq A$, the ball centered in $f$ of radius $\alpha$ is defined as usual: $B(f, \alpha):=\{g \in X \mid \delta(f, g) \subseteq \alpha\}$. In 1 a space $(X, \delta)$ is said to be pairwise complete if, for each $f, g \in X$ and $\alpha, \beta \subseteq A$,

$$
B(f, \alpha \cup \beta)=B(g, \alpha \cup \beta) \text { implies } B(f, \alpha) \cap B(g, \beta) \neq \emptyset .
$$

This property is easily seen to be equivalent to:

$$
\delta(f, g) \subseteq \alpha \cup \beta \text { implies } \delta(f, h) \subseteq \alpha \text { and } \delta(h, g) \subseteq \beta, \text { for some } h \in X .
$$

Recall also from [1] that a space is said to be spherically complete if the intersection $\bigcap_{i \in I} B\left(f_{i}, \alpha_{i}\right)$ of every chain $\left\{B\left(f_{i}, \alpha_{i}\right) \mid i \in I\right\}$ of balls is non-empty. It was shown in [20] that, when $A$ is finite, every space over $P(A)$ is spherically complete. To stress the importance of these two conditions, pairwise completeness and spherical completeness, let us recall the following result from [1].

Proposition 25. In the category of spaces, the injective objects are the spaces that are both pairwise complete and spherically complete.

If $\left(X, \delta_{X}\right)$ is a space and $Y \subseteq X$, then the restriction of $\delta_{X}$ to $Y$ induces a space $\left(Y, \delta_{X}\right)$; we say then that $\left(Y, \delta_{X}\right)$ is a subspace of $X$. Notice that the inclusion of $Y$ into $X$ yields an isometry of spaces.

Our main example of space over $P(A)$ is $\left(D^{A}, \delta\right)$, with $D^{A}$ the set of functions from $A$ to $D$ and the distance defined by

$$
\delta(f, g):=\{a \in A \mid f(a) \neq g(a)\} .
$$

A second example is a slight generalization of the previous one. Given a surjective function $\pi: E \longrightarrow A$, let $\operatorname{Sec}(\pi)$ denote the set of all sections of $\pi$, that is the functions $f: A \longrightarrow E$ such that $\pi \circ f=i d_{A}$; the formula in (3) also defines a distance on $\operatorname{Sec}(\pi)$. Clearly, $(\operatorname{Sec}(\pi), \delta)$ is a subspace of $\left(E^{A}, \delta\right)$ and, considering the first projection $\pi_{1}: A \times D \longrightarrow A$, we can see that $\left(D^{A}, \delta\right)$ is isomorphic to the space $\left(\operatorname{Sec}\left(\pi_{1}\right), \delta\right)$. By identifying $f \in \operatorname{Sec}(\pi)$ with the vector $\left\langle f_{a} \in \pi^{-1}(a) \mid a \in A\right\rangle$, we see that

$$
\operatorname{Sec}(\pi)=\prod_{a \in A} E_{a}, \quad \text { where } E_{a}:=\pi^{-1}(a) .
$$

That is, the underlying set of a space $(\operatorname{Sec}(\pi), \delta)$ is that of a universal $\mathrm{S} 5^{A}$-product frame. Our next observations are meant to identify the particular role of the universal $\mathrm{S} 5{ }^{A}$-product frames as spaces.

Proposition 26. Every space of the form $(\operatorname{Sec}(\pi), \delta)$ is both pairwise complete and spherically complete.

Proof. It is immediate to verify that $(\operatorname{Sec}(\pi), \delta)$ is pairwise complete. For spherical completeness, let $\mathcal{C}:=\left\{B\left(f_{i}, \alpha_{i}\right) \mid i \in I\right\}$ be a chain of balls. For each $a \in A$ pick $*_{a} \in D_{a}$ and define $f$ as follows:

$$
f(a)= \begin{cases}f_{i}(a), & \text { if } a \notin \alpha_{i} \text { for some } i \in I, \\ *_{a}, & \text { otherwise. }\end{cases}
$$

\footnotetext{
${ }^{1}$ As $P(A)$ is not totally ordered, we avoid calling a morphism "non expanding map" as it is often done in the literature.
} 
Let us show that $f$ is well defined. Namely, suppose that $a \notin \alpha_{i}$ and $a \notin \alpha_{j}$. Since $\mathcal{C}$ is a chain, we can suppose, without loss of generality, that $B\left(f_{i}, \alpha_{i}\right) \subseteq B\left(f_{j}, \alpha_{j}\right)$ so $\delta\left(f_{i}, f_{j}\right) \subseteq \alpha_{j}$. Since $a \notin \alpha_{j}$ it follows that $f_{i}(a)=f_{j}(a)$.

Let now $i \in I$ be arbitrary; if $a \notin \alpha_{i}$, then $f(a)=f_{i}(a)$, so $\delta\left(f, f_{i}\right) \subseteq \alpha_{i}$ and $f \in B\left(f_{i}, \alpha_{i}\right)$. It follows that $f \in \bigcap_{i \in I} B\left(f, \alpha_{i}\right)$.

Theorem 27. Every space $(X, \delta)$ over $P(A)$ has an isometry into some space of the form $(\operatorname{Sec}(\pi), \delta)$. If $(X, \delta)$ is pairwise and spherically complete, then this isometry is an isomorphism.

Proof. For each $a \in A$, let $D_{a}=\{B(f, A \backslash\{a\}) \mid f \in X\}$. That is, $D_{a}$ is the quotient of $X$ by the equivalence relation identifying $f$ and $g$ when $\delta(f, g) \subseteq A \backslash\{a\}$. Let $\pi: \sum_{a \in A} D_{a} \longrightarrow A$ be the obvious projection.

We associate to $f \in X$ the vector $\psi(f)=\langle B(f, A \backslash\{a\}) \mid a \in A\rangle$. Let us argue that the correpondence $\psi$ is an isometry:

$$
\begin{aligned}
a \notin \delta(\psi(f), \psi(g)) & \text { iff } B(f, A \backslash\{a\})=B(g, A \backslash\{a\}) \\
& \text { iff } \delta(f, g) \subseteq A \backslash\{a\} \text { iff } a \notin \delta(f, g),
\end{aligned}
$$

thus $\delta(\psi(f), \psi(g))=\delta(f, g)$. In particular, when the space is reduced (i.e. $\delta(f, g)=$ $\emptyset$ implies $f=g), \psi$ is an injective map.

Next, we suppose that $(X, \delta)$ is pairwise and spherically complete and argue that $\psi$ is surjective. To this goal, we fix a well-ordering on $A$, say $A=\left\{a_{\lambda} \mid \lambda<\tau\right\}$. For $\lambda<\tau$, let us also set $A_{\lambda^{-}}:=\left\{a_{\beta} \in A \mid \beta \leq \lambda\right\}$ and $A_{\lambda^{+}}:=A \backslash A_{\lambda^{-}}=\left\{a_{\beta} \in\right.$ $A \mid \beta>\lambda\}$.

Let $v:=\left\langle B\left(f_{\lambda}, A \backslash\left\{a_{\lambda}\right\}\right) \mid \lambda<\tau\right\rangle \in \operatorname{Sec}(\pi)$; we need to construct a preimage of $v$ by $\psi$. To this end, we construct, by induction on $\lambda<\tau$, a family $\left\{g_{\lambda} \in X \mid \lambda<\tau\right\}$ such that $g_{\lambda} \in B\left(g_{\beta}, A_{\beta}\right)$ for $\beta \leq \lambda$ and $\delta\left(g_{\lambda}, f_{\lambda}\right) \subseteq A \backslash\left\{a_{\lambda}\right\}$. Let $\lambda<\tau$ be an ordinal and suppose that we have defined $g_{\beta}$ with these properties for each $\beta<\lambda$. As $\left\{B\left(g_{\beta}, A_{\beta^{+}}\right) \mid \beta<\lambda\right\}$ is a chain, we can pick $g \in \bigcap_{\beta<\lambda} B\left(g_{\beta}, A_{\beta^{+}}\right)$. Notice that if $\lambda=\gamma+1$ is a successor cardinal, then we can simply pick $g_{\gamma}$.

We use pairwise completeness to define $g_{\lambda}$ as some $h$ with $\delta(g, h) \subseteq\left\{a_{\lambda}\right\}$ and $\delta\left(h, f_{\lambda}\right) \subseteq \delta\left(g, f_{\lambda}\right) \backslash\left\{a_{\lambda}\right\}$ (if $a_{\lambda} \notin \delta\left(g, f_{\lambda}\right)$, then we can take $h=g$ ). Clearly, $\delta\left(g_{\lambda}, g_{\lambda}\right)=\emptyset \subseteq A_{\lambda^{+}}$and, for $\beta<\lambda$, we have $\delta\left(g_{\lambda}, g_{\beta}\right) \subseteq \delta\left(g_{\lambda}, g\right) \cup \delta\left(g, g_{\beta}\right)=$ $\left\{a_{\lambda}\right\} \cup \delta\left(g, g_{\beta}\right) \subseteq\left\{a_{\lambda}\right\} \cup A_{\beta} \subseteq A_{\beta^{+}}$.

Let now $g \in \bigcap_{\lambda<\tau} B\left(g_{\beta}, A_{\beta^{+}}\right)$. If $\lambda<\tau$, then

$$
\delta\left(g, f_{\lambda}\right) \subseteq \delta\left(g, g_{\lambda}\right) \cup \delta\left(g_{\lambda}, f_{\lambda}\right) \subseteq A_{\lambda^{+}} \cup\left(A \backslash\left\{a_{\lambda}\right\}\right) \subseteq A \backslash\left\{a_{\lambda}\right\} .
$$

This shows that $B\left(g, A \backslash\left\{a_{\lambda}\right\}\right)=B\left(f_{\lambda}, A \backslash\left\{a_{\lambda}\right\}\right)$ or, stated otherwise, $\psi(g)_{\lambda}=v_{\lambda}$, for each $\lambda<\tau$, so $g$ is a preimage of $v$.

By the last two Propositions and the last Theorem, we obtain:

Corollary 28. Universal product frames are, up to isomorphism, the injective objects in the category of spaces.

Continuous supbspaces and completeness. In the following, let $\left(X, \delta_{X}\right)$ be a fixed pairwise complete space. Our next goal is to devise criteria to recognize pairwise complete subspaces of $\left(X, \delta_{X}\right)$. To this end, let us introduce the notion of continuous subspaces of $\left(X, \delta_{X}\right)$ as follows: for a subspace $Y$ of $X$, we define

$$
\nu_{Y}(f):=\bigcap\{\delta(f, g) \mid g \in Y\}
$$


and say that a subspace $Y$ of $X$ is continuous if, for each $f \in X, \nu_{Y}(f)=\emptyset$ implies $f \in Y$.

Lemma 29. A continuous subspace $Y$ of $X$ is pairwise complete.

Proof. Let $f, g \in Y$ with $\delta(f, g) \subseteq \alpha \cup \beta$. Let $h \in X$ be such that $\delta(f, h) \subseteq \alpha \backslash \beta$ and $\delta(h, g) \subseteq \beta$; then $\bigcap_{k \in Y} \delta(h, k) \subseteq \delta(h, f) \cap \delta(h, g) \subseteq \alpha \backslash \beta \cap \beta=\emptyset$, so $h \in Y$ since $Y$ is continuous.

Lemma 30. If $X$ is also spherically complete, then a continuous subspace $Y$ of $X$ is pairwise and spherically complete.

Proof. By Theorem 27, we can suppose that $X$ is of the form $\prod_{a \in A} E_{a}$. A subset $Y$ of $X$ is then continuous if $f \in Y$, whenever for all $a \in A$ there exists $g \in Y$ with $f(a)=g(a)$. Let us put $Y_{a}:=\{g(a) \mid g \in Y\}$, so $Y \subseteq \prod_{a \in A} Y_{a}$. If $f \in \prod_{a \in A} Y_{a}$, then for each $a \in A$ there is some $g \in G$ such that $f(a)=g(a)$ and therefore $f \in Y$. We have therefore $Y=\prod_{a \in A} X_{a}$, so $Y$ is pairwise and spherically complete.

Lemma 31. If $Y$ is pairwise complete subspace of $X$, then, for each $f \in X$, the set $\{\alpha \subseteq A \mid B(f, \alpha) \cap Y \neq \emptyset\}$ is closed under finite intersections. Consequently, if $Y$ is spherically complete, then, for each $f \in X$, there exists $g \in Y$ with $\delta(f, g)=\nu_{Y}(f)$.

Proof. Let $\mathcal{A}$ be the set of all $\alpha \subseteq A$ such that $B(f, \alpha) \cap Y \neq \emptyset$. For each $\alpha \in \mathcal{A}$, choose $t_{\alpha} \in B(f, \alpha) \cap Y$.

Observe that, for $\alpha, \alpha^{\prime} \in \mathcal{A}, \delta\left(t_{\alpha}, t_{\alpha^{\prime}}\right) \subseteq \delta\left(t_{\alpha}, f\right) \cup \delta\left(f, t_{\alpha^{\prime}}\right) \subseteq \alpha \cup \alpha^{\prime}$. That is, the function $t$ sending $\alpha$ to $t_{\alpha}$ is a $\gamma$-Cauchy function to $Y$ as defined in 1, Definition 2.8], where $\gamma=\bigcap \mathcal{A}$.

Observe next that $\delta\left(t_{\alpha}, t_{\alpha^{\prime}}\right) \subseteq \alpha \cup \alpha^{\prime}$ so, by pairwise completeness of $Y, \delta\left(t_{\alpha}, h\right) \subseteq$ $\alpha$ and $\delta\left(h, t_{\alpha^{\prime}}\right) \subseteq \alpha^{\prime}$ for some $h \in Y$. It follows that $\delta(f, h) \subseteq \alpha \cap \alpha^{\prime}$, showing that $\alpha \cap \alpha^{\prime} \in \mathcal{A}$. In particular, $t$ is a $\gamma$-Cauchy net, as defined in [1, Definition 2.9].

If we assume that $Y$ is spherically complete, then we can use Proposition 2.16 in [1] to deduce that, for some $g \in Y, \delta\left(g, t_{\alpha}\right) \subseteq \alpha$, for each $\alpha \in \mathcal{A}$. For such a $g \in Y$, we have $\delta(f, g) \subseteq \delta\left(f, t_{\alpha}\right) \cup \delta\left(t_{\alpha}, g\right) \subseteq \alpha$, showing that $\delta(f, g) \subseteq \nu_{Y}(f)$. As $g \in Y$, we also have $\nu_{Y}(f) \subseteq \delta(f, g)$ and $\nu_{Y}(f)=\delta(f, g)$.

Corollary 32. If $Y$ is a pairwise complete and spherically complete subspace of $X$, then $Y$ is a continuous subspace of $X$.

Proof. Let $f \in X$ be such that $\nu_{Y}(f)=\bigcap_{g \in Y} \delta(f, g)=\emptyset$. By Lemma 31, let $h \in Y$ such that $\nu_{Y}(f)=\delta(f, h)$, so $\delta(f, h)=\emptyset$ and $f=h \in Y$.

Corollary 33. If $X$ is also spherically complete, then a subspace of $X$ is continuous if and only if it is pairwise and spherically complete.

Let us remark that when $A$ is finite, then $\left(X, \delta_{X}\right)$ and all its subspaces are spherically complete, so in this case continuity and pairwise completeness are equivalent conditions.

Modules. We say that a function $v: X \longrightarrow P(A)$ is a module if

$$
v(f) \subseteq \delta(f, g) \cup v(g) .
$$

In (enriched) category theory "module" is a standard naming for an enriched functor from an enriched category to the base category enriched on itself. Here a module 
can be seen as a space morphism from $(X, \delta)$ to the space $(P(A), \Delta)$, where $\Delta$ is the symmetric difference.

We let Modules $(X)$ be the set of all modules $v$; we order this set by letting $v \leq w$ if and only if $w(f) \subseteq v(f)$, for each $f \in Y$ (that is, we take the reverse pointwise order). Let use $S u b(X)$ for the set of subspaces of $X$, ordered by inclusion - thus $\operatorname{Sub}(X)$ is the usual power set of $X$. Given a module $v$, let us define

$$
\mathrm{S}_{v}:=\{x \in X \mid v(x)=\emptyset\} .
$$

It is easily seen that $\mathrm{S}: \operatorname{Modules}(X) \longrightarrow S u b(X)$, sending $v$ to $\mathrm{S}_{v}$, is also orderpreserving.

Lemma 34. The map $\nu$ is left adjoint to $\mathrm{S}$.

Proof. As both maps are order-preserving, we shall show that the usual unit and counit laws hold. If $f \in Y$, then $\nu_{Y}(f) \subseteq \delta(f, f)=\emptyset$; thus $Y \subseteq \mathrm{S}_{\nu_{Y}}$. Let us argue for the counit law. For each $f \in X$ and $g \in \mathrm{S}_{v}$-i.e. when $v(g)=\emptyset$-we have $v(f) \subseteq \delta(f, g) \cup v(g)=\delta(f, g)$. It follows that $v(f) \subseteq \nu_{\mathrm{S}_{v}}(f)$, for each $f \in X$. This means that $\nu_{\mathrm{S}_{v}} \leq v$ in Modules $(X)$.

Lemma 35. For each module $v, \mathrm{~S}_{v}$ is a continuous subspace of $X$.

Proof. We already observed that $v(f) \subseteq \nu_{\mathrm{S}_{v}}(f)$, that is, $v(f) \subseteq \bigcap_{g \in \mathrm{S}_{v}} \delta(f, g)$. If the latter expression is equal to the emptyset, then $v(f)=\emptyset$, whence $f \in \mathrm{S}_{v}$. This shows that $\mathrm{S}_{v}$ is a continuous subspace of $X$.

Proposition 36. A subspace $Y$ of $X$ is continuous if and only if $\mathrm{S}_{\nu_{Y}}=Y$. Thus, for any $Y \subseteq X, \mathrm{~S}_{\nu_{Y}} \subseteq X$ is the least continuous subspace of $X$ containing $Y$.

Proof. Observe first that if $Y=\mathrm{S}_{\nu_{Y}}$, then $Y$ is continuous by Lemma 35 .

Conversely, let us suppose that $Y$ is continuous. By adjointness, $Y \subseteq \mathrm{S}_{\nu_{Y}}$ holds, so we argue for the reverse inclusion. If $f \in \mathrm{S}_{\nu_{Y}}$, then $\emptyset=\nu_{Y}(f)=\bigcap_{g \in Y} \delta(f, g)$, so $f \in Y$. Therefore $\mathrm{S}_{\nu_{Y}} \subseteq Y$.

The last statement follows from the characterization of the continuous subspaces of $X$ as the fixed-points of the closure operator $\mathrm{S}_{\nu(-)}$.

Proposition 37. A module $v$ is such that $\nu_{\mathrm{S}_{v}}=v$ if and only if either $v(f)=A$, for each $f \in X$, or $v(f)=\emptyset$, for some $f \in X$.

Proof. If $v(f)=A$ for each $f \in X$, then $S_{v}=\emptyset$ (since we are assuming that $A \neq \emptyset$ ). It follows that $\nu_{\mathrm{S}_{v}}(f)=A$ for each $f \in X$ and $\nu_{\mathrm{S}_{v}}=v$.

Suppose now that $v(g)=\emptyset$ for some $g \in X$. By adjointness, we have $v(f) \subseteq$ $\nu_{\mathrm{S}_{v}}(f)$, for all $f \in X$; thus we need to argue for the opposite inclusion. Fix $f \in X$; we exhibit next $h \in X$ such that $v(h)=\emptyset$ and $\delta(f, h) \subseteq v(f)$. It shall follow that $\nu_{\mathrm{S}_{v}}(f)=\bigcap_{v(h)=\emptyset} \delta(f, h) \subseteq v(f)$. Since $v(f) \subseteq \delta(f, g) \cup v(g)=\delta(f, g)$, we can write $\delta(f, g)=v(f) \cup(\delta(f, g) \backslash v(f))$. We use now pairwise completeness to pick $h \in X$ such $\delta(f, h) \subseteq v(f)$ and $\delta(h, g) \subseteq \delta(f, g) \backslash v(f)$. Then $v(h) \subseteq$ $\delta(h, f) \cup v(f) \subseteq v(f)$, and $v(h) \subseteq \delta(h, g) \cup v(g)=\delta(h, g) \subseteq \delta(f, g) \backslash v(f)$. It follows that $v(h) \subseteq v(f) \cap A \backslash v(f)$, whence $v(h)=\emptyset$.

For the converse direction, suppose that $v(f)=\nu_{\mathrm{S}_{v}}(f)$ for each $f \in X$. If $v(f) \neq \emptyset$, for each $f \in Y$, then $v(f)=\nu_{\mathrm{S}_{v}}(f)=A$, for each $f \in X$. Otherwise, $v(f)=\emptyset$, for some $f \in X$. 
Remark 38. Proposition 36 characterizing continuous subspaces as closed subsets of a closure operator, suggests that pairwise complete spaces might have some algebraic nature as well. This is actually the case. It is easily verified that a space $(X, \delta)$ is pairwise complete if and only if, for each $\alpha, \beta$ such that $\alpha \cap \beta=\emptyset$, and for each $f, g \in X, \delta(f, g) \subseteq \alpha \cup \beta$ implies $\delta(f, h) \subseteq \alpha$ and $\delta(h, g) \subseteq \beta$ for some $h \in X$. We observe that such an $h$ is unique. Suppose that $\alpha \cap \beta=\emptyset$ and let $h_{i}, i=1,2$ with $\delta\left(f, h_{i}\right) \subseteq \alpha$ and $\delta\left(h_{i}, g\right) \subseteq \beta$. Then $\delta\left(h_{1}, h_{2}\right) \subseteq \delta\left(h_{1}, f\right) \cup \delta\left(f, h_{2}\right) \subseteq \alpha$ and similarly $\delta\left(h_{1}, h_{2}\right) \subseteq \beta$. It follows that $\delta\left(h_{1}, h_{2}\right) \subseteq \alpha \cap \beta=\emptyset$ and $h_{1}=h_{2}$.

Pairwise complete spaces and universal product frames. We already observed, in the display (4), that the underlying set of a space of the form $(\operatorname{Sec}(\pi), \delta)$ with $\pi: E \longrightarrow A$ is that of a universal $\mathrm{S} 5^{A}$-product frame. Something more is true: we can define the transition relations of the universal $\mathrm{S} 5^{A}$-product frame by means of the metric. Indeed, for each $a \in A$, we have

$$
f R_{a} g \text { iff } \delta(f, g) \subseteq\{a\} .
$$

On the other hand, if $A$ is finite, then the metric is completely determined from the transition relation of the frame, using the notion of $\alpha$-path introduced in Section 2 as follows:

$$
\delta(f, g)=\bigcap\{\alpha \subseteq A \mid \text { there exists an } \alpha \text {-path from } f \text { to } g\} .
$$

We cast our observations in a Proposition:

Proposition 39. If $A$ is finite, then there is a bijective correspondence bewtween spaces over $P(A)$ of the form $(\operatorname{Sec}(\pi), \delta)$ and universal $\mathrm{S} 5^{A}$-product frames.

Pairwise complete spaces and lattices. We can generalize the construction of the relational lattice $\mathrm{R}(D, A)$ starting from an arbitrary space $(X, \delta)$. We say that a subset $Z \subseteq A \cup X$ is closed if $x \in Z$ whenever $\delta(x, y) \subseteq Z \cap A$ and $y \in Z$. The set of closed subsets of $A \cup X$ is then a Moore family.

Definition 40. The lattice $\mathcal{L}(X, \delta)$ is the lattice of closed subsets of $A \cup X$.

Obviously, $\mathcal{L}\left(D^{A}, \delta\right)$ is the relational lattice $\mathrm{R}(D, A)$. The lattice $\mathcal{L}(X, \delta)$ can be shown to be pluperfect when $(X, \delta)$ is reduced and symmetric. Yet, for the sake of the undecidability result, we shall only need that $\mathcal{L}(X, \delta)$ is an atomistic pluperfect lattice when $A$ is finite and $(X, \delta)$ is pairwise complete.

Proposition 41. If $A$ is finite and $(X, \delta)$ is pairwise complete, then $\mathcal{L}(X, \delta)$ is isomorphic to the lattice $\mathrm{L}(\mathfrak{U})$ for some universal $\mathrm{S} 5^{A}$-product frame $\mathfrak{U}$.

Proof. By Theorem 27 the space $(X, \delta)$ is isomorphic to the space $(\operatorname{Sec}(\pi), \delta)$, for some surjective $\pi: E \longrightarrow A$. The construction $\mathcal{L}$ clearly sends isomorphic spaces to isomorphic to isomorphic lattices. Therefore, we assume that $(X, \delta)=(\operatorname{Sec}(\pi), \delta)$ and prove that $\mathcal{L}(X, \delta)=\mathrm{L}(\mathfrak{U})$. We have $X=\prod_{a \in A} E_{a}$ with $E_{a}=\pi^{-1}(a)$, while $\delta(f, g)=\{a \in A \mid f(a) \neq g(a)\}$. It is easily verified that $\delta(f, g) \subseteq \alpha$ if and only if there is an $\alpha$-path from $f$ to $g$ in the universal $S 5^{A}$ product frame $\mathfrak{U}$ on $\prod_{a \in A} E_{a}$. Therefore, the two Moore families, $\mathcal{L}(X, \delta)$ and $\mathrm{L}(\mathfrak{U})$, are the same.

From the above theorem and from the preliminary investigation of the structure of the lattices $\mathrm{L}(\mathfrak{F})$ in Section 5 , we can infer the following statement. 
Corollary 42. If $A$ is finite and $(X, \delta)$ is pairwise complete, then $\mathcal{L}(X, \delta)$ is an atomistic pluperfect lattice, where the set of join-irreducible elements can be identified with $A \cup X$, every element $a \in A$ is join-prime, and minimal join-covers of $f \in X$ are of the form

$$
f \triangleleft_{\mathrm{m}} \delta(f, g) \cup\{g\}
$$

for each $g \in X$.

Proof. The statement follows from Propositon 22 and from the observation that an $\alpha$-path from $f$ to $g$ is minimal if and only if $\alpha=\delta(f, g)$.

Let us remark that the above statement holds even when $A$ is not finite or when $(X, \delta)$ is not pairwise complete. In particular, if $A$ is infinite and the universal $\mathrm{S} 5^{A}$-product frame $\mathfrak{U}$ has $(\operatorname{Sec}(\pi), \delta)$ as underlying space, then the lattice $\mathrm{L}(\mathfrak{U})$ defined in Section 5 and the lattice $\mathcal{L}(\operatorname{Sec}(\pi), \delta)$ defined here need not to be equal. For instance, if $\delta(f, g)$ is an infinite set, then $\delta(f, g) \cup\{g\}$ is an infinite minimal join-cover of $f$, while we observed before that any minimal join-cover in $\mathrm{L}(\mathfrak{U})$ is finite.

\section{Principal ideals and filters in Relational Lattices}

The purpose of this Section is to prove the following statement.

Theorem 43. If $L$ is a finite subdirectly-irreducible atomistic lattice which has a lattice embedding into some relational lattice $\mathrm{R}(D, A)$, then there exists an embeddings of $L$ into some other relational lattice $\mathrm{R}(D, B)$ which moreover preserves $\perp$ and $T$.

The theorem is an immediate consequence of Propositions 44] and 46 that follows. These propositions mainly deal with the structure of principal ideals and filters in a relational lattice $\mathrm{R}(D, A)$, namely the sublattices of the form $\downarrow Z:=\{W \in \mathrm{R}(D, A) \mid$ $W \subseteq Z\}$ and $\uparrow Z:=\{W \in \mathrm{R}(D, A) \mid Z \subseteq W\}$.

In the proofs of these propositions we use the isomorphism between $P\left(A \sqcup D^{A}\right)$ and $P(A) \times P\left(D^{A}\right)$ to represent the lattice $\mathrm{R}(D, A)$ as the set of pairs $(\alpha, Y)$ with $\alpha \subseteq A$ and $Y \subseteq D^{A} \alpha$-closed.

Proposition 44. If $L$ is a subdirectly-irreducible lattice which has an embedding $i: L \longrightarrow \mathrm{R}(D, A)$, then there is a subset $\alpha \subseteq A$ and an embedding $j: L \longrightarrow \mathrm{R}(D, \alpha)$ that preserves $\mathrm{T}$.

Proof. Suppose $i(\top) \neq\left(A, D^{A}\right)$, say $i(\top)=(\alpha, Y)$, with $Y \alpha$-closed. Call $M$ the ideal $\downarrow(\alpha, Y)$, so $L$ embeds into $M$ while preserving $\top$. Let us study the structure of $M$. This lattice is clearly atomistic and pluperfect by Lemma 3. Its set of atoms is $\alpha \cup Y$, while the minimal join-covers are of the form $f \triangleleft_{\mathrm{m}} \delta(f, g) \cup\{g\}$ whenever $f, g \in Y$ and $\delta(f, g) \subseteq \alpha$.

Notice now that if $f \in Y$, then the ball $B(f, \alpha)$ is contained in $Y$, since $Y$ is $\alpha$-closed. This implies that $A_{f}:=\alpha \cup B(f, \alpha)$ is a $D$-closed subset of $\mathcal{J}_{c}(M)$ and, $M_{A_{f}}$ defined in Lemma 4, is a lattice quotient of $M$. We notice that the OD-graph of $M_{A_{f}}$ is isomorphic to the one of $\mathrm{R}(D, \alpha)$, so $M_{A_{f}}$ itself is isomorphic to $\mathrm{R}(D, \alpha)$.

Since moreover $\bigcup_{f \in Y} \alpha \cup B(f, \alpha)=\alpha \cup Y$, then $\left\langle\pi_{A_{f}} \mid f \in Y\right\rangle: M \longrightarrow$ $\prod_{f \in Y} M_{A_{f}}$ is, by Lemma 5, a subdirect decomposition of $M$. Therefore $L$ embeds into $\prod_{f \in Y} M_{A_{f}}$ and since $L$ is subdirectly-irreducible, it embeds into some 
$M_{A_{f}}$ and such embedding preserves $T$. Since $M_{A_{f}}$ is isomorphic to $\mathrm{R}(D, \alpha)$, we conclude that $L$ embeds into $\mathrm{R}(D, \alpha)$ while preserving $\mathrm{T}$.

For $B \subseteq A$, let us define $\psi_{A, B}: P(A) \times P\left(D^{A}\right) \longrightarrow P(B) \times P\left(D^{B}\right)$ by the following formula:

$$
\psi_{A, B}(\alpha, X):=\left(\alpha \cap B, X \|_{B}\right) .
$$

Lemma 45. The map $\psi_{A, B}$ restricts to an order-preserving map from $\mathrm{R}(D, A)$ to $\mathrm{R}(D, B)$. Its further restriction to the filter $\uparrow\left(B^{\mathrm{c}}, \emptyset\right) \subseteq \mathrm{R}(D, A)$ yields an isomorphism with $\mathrm{R}(D, B)$.

Proof. We suppose that $X$ is $\alpha$-closed and argue $X \|_{B}$ is $\alpha \cap B$-closed. If $g \in X$, $f \in D^{B}$, and $\delta_{D^{B}}\left(f, g_{\uparrow_{B}}\right) \subseteq \alpha \cap B$, then we can extend $f$ to $f^{\prime} \in D^{A}$, so $f^{\prime}{{ }_{B}}_{B}=f$ and $f^{\prime}(x)=g(x)$ for all $x \in B^{\mathrm{c}}$. It follows that $\delta_{D^{A}}\left(f^{\prime}, g\right)=\delta_{D^{B}}\left(f, g_{\uparrow_{B}}\right) \subseteq \alpha \cap B$, so $f^{\prime} \in X$ since $X$ is $\alpha$-closed. Then $f=f^{\prime} \uparrow_{B} \in X \|_{B}$.

We argue similarly that if $(\beta, Y) \in \mathrm{R}(D, B)$, then $\left(\beta \cup B^{\mathrm{c}}, i_{A}(Y)\right)$ belongs to $\mathrm{R}(D, A)$, namely that $i_{A}(Y)$ is $\beta \cup B^{\mathrm{c}}$-closed when $Y$ is $\beta$-closed. Let $f \in D^{A}$ and $g \in i_{A}(Y)$ be such that $\delta(f, g) \subseteq \beta \cup B^{\mathrm{c}}$. Now $\delta\left(f_{\uparrow_{B}}, g_{\uparrow_{B}}\right) \subseteq\left(\beta \cup B^{\mathrm{c}}\right) \cap B=\beta$, and since $g_{\uparrow_{B}} \in Y$ and $Y$ is $\beta$-closed, we have $f_{\uparrow_{B}} \in Y$, that is $f \in i_{A}(Y)$.

Observe moreover that $\left(\alpha \cap B, X \|_{B}\right) \subseteq(\beta, Y)$ holds if and only if $(\alpha, X) \subseteq$ $\left(\beta \cup B^{\mathrm{c}}, i_{A}(Y)\right)$, so the two maps are adjoints to each other, in particular they are monotonic.

Next $\psi_{A, B}\left(\beta \cup B^{\mathrm{c}}, i_{A}(Y)\right)=\left(\left(\beta \cup B^{\mathrm{c}}\right) \cap B, i_{A}(Y) \|_{B}\right)=(\beta, Y)$, thus $\psi_{A, B}$ is surjective. Finally, let us argue that $\psi_{A, B}$ is injective if restricted to $\uparrow\left(B^{\mathrm{c}}, \emptyset\right)$. Let $(\alpha, X),\left(\alpha^{\prime}, X^{\prime}\right) \in \mathrm{R}(D, A)$ with $B^{\mathrm{c}} \subseteq \alpha \cap \alpha^{\prime}$ and $\psi_{A, B}(\alpha, X)=\psi_{A, B}\left(\alpha^{\prime}, X^{\prime}\right)$. Then $\alpha \cap B=\alpha^{\prime} \cap B, \alpha=(\alpha \cap B) \cup B^{\mathrm{c}}$, and $\alpha^{\prime}=\left(\alpha^{\prime} \cap B\right) \cup B^{\mathrm{c}}$, imply $\alpha=\alpha^{\prime}$. Let $f \in X$, so $f_{\uparrow_{B}} \in X\left\|_{B}=X^{\prime}\right\|_{B}$, so there exists $f^{\prime} \in X^{\prime}$ with $f_{\uparrow_{B}}^{\prime}=f_{\uparrow_{B}}$. Since $X^{\prime}$ is $B^{\mathrm{c}}$ closed and $\delta\left(f, f^{\prime}\right) \subseteq B^{\mathrm{c}}$ we have $f \in X^{\prime}$. Thus we have $X \subseteq X^{\prime}$; a similar argument yields $X^{\prime} \subseteq X$, so $X=X^{\prime}$.

Proposition 46. If a finite subdirectly-irreducible atomistic lattice $L$ has a $T$ preserving lattice embedding $i: L \longrightarrow \mathrm{R}(D, A)$, then there exists an embedding $j: L \longrightarrow \mathrm{R}(D, B)$ which preserves $\top$ and $\perp$.

Proof. By Lemma 45. if $i(\perp)=\left(B^{\mathrm{c}}, X\right)$ for some $B \subseteq A$, then $j=\psi_{A, B} \circ i: L \longrightarrow$ $\mathrm{R}(D, B)$ is an embedding which preserves $\top$ and such that $j(\perp)=(\emptyset, Y)$ for some $Y \subseteq D^{B}$.

Suppose now that $Y \neq \emptyset$. Let $\mu: \mathrm{R}(D, B) \longrightarrow L$ be left adjoint to $j$, so $\mu$ is surjective and, moreover, each atom $a \in \mathcal{J}_{\mathrm{c}}(L)$ has some $k \in B \cup D^{B}$ with $\mu(k)=a$. Notice also that $\mu(k)=\perp$ if and only if $k \in Y$, for each $k \in B \cup D^{B}$. Let us argue that every element of $\mathcal{J}_{\mathrm{c}}(L)$ is join-prime. Let $a \in \mathcal{J}_{\mathrm{c}}(L)$ and pick $k \in B \cup D^{B}$ such that $\mu(k)=a$. If $k \in B$, then $a=\mu(k)$ is join-prime, since $\mu$ sends a join-prime element either to a join-prime element or to $\perp$. Suppose now $k=f \in D^{B}$ and recall that $\mu(f)=a \neq \perp$ implies $f \notin Y$. Pick $g \in Y$, so $f \leq \bigvee \delta(f, g) \vee g$ and $a=\mu(f) \leq \bigvee \mu(\delta(f, g)) \vee \mu(g)=\bigvee \mu(\delta(f, g))$. Since $\mu$ sends join-prime elements to join-prime elements or to $\perp$, we see that $a$ has a join-cover made up of join-prime elements only. Lemma 7 implies then that $a$ is join-prime.

We have argued that either $Y=\emptyset$, so $j$ preserves $\perp$; or $Y \neq \emptyset$, in which case all the elements of $\mathcal{J}_{c}(L)$ are join-prime and atoms. In the last case, however, $L$ is a two elements Boolean algebra, since $L$ is subdirectly-irreducible and distributive. 
Such an algebra can obviously be embedded into a relational lattice while preserving $\top$ and $\perp$.

\section{From lattice Embeddings to SURJECTIVE $p$-MORPhisms}

We prove in this Section the converse of Theorem 20

Theorem 47. Let $A$ be a finite set, let $\mathfrak{F}$ be a finite rooted full $\mathrm{S} 4$ A-frame. If $\mathrm{L}(\mathfrak{F})$ embeds into a relational lattice $\mathrm{R}(D, B)$, then there exists a universal $\mathrm{S} 5^{A}$-product frame $\mathfrak{U}$ and a surjective p-morphism from $\mathfrak{U}$ to $\mathfrak{F}$.

To prove the Theorem, we study bound-preserving embeddings of finite atomistic lattices into lattices of the form $\mathrm{R}(D, B)$. Let in the following

$$
i: L \longrightarrow \mathrm{R}(D, B)
$$

be a fixed bound-preserving lattice embedding, with $L$ a finite atomistic lattice. Since $L$ is finite, $i$ has a left adjoint $\mu: \mathrm{R}(D, B) \longrightarrow L$. By abuse of notation, we shall also use the same letter $\mu$ to denote the restriction of this left adjoint to the set of completely join-irreducible elements of $\mathrm{R}(D, B)$ which, we recall, is identified with $B \cup D^{B}$. It is a general fact - and the main ingredient of Birkhoff's duality for finite distributive lattices - that left adjoints to bound-preserving lattice morphism preserve join-prime elements. Thus we have:

Lemma 48. If $b \in B$, then $\mu(b)$ is join-prime.

Proof. Suppose $b \in B$ and $\mu(b) \leq \bigvee X$. Then $b \leq i(\bigvee X)=\bigvee i(X)$, so $b \leq i(x)$ for some $x \in X$, since $b$ is join-prime. It follows that $\mu(b) \leq x$, for some $x \in X$.

It is not in general true that left adjoints send join-irreducible elements to joinirreducible elements, and this is a main difficulty towards a proof of Theorem 47 Yet, the following statements hold:

Lemma 49. For each $x \in \mathcal{J}_{\mathrm{c}}(L)$ there exists $y \in B \cup D^{B}$ such that $\mu(y)=x$.

Proof. Since $i$ is an embedding, then its left adjoint $\mu$ is surjective. So if $x \in \mathcal{J}_{\mathrm{c}}(L)$, then there exists $y \in \mathrm{R}(D, B)$ with $\mu(y)=x$. Write $y=\bigvee_{i \in I} z_{i}$ with each $z_{i} \in$ $B \cup D^{B}$. Then $x=\bigvee_{i \in I} \mu\left(z_{i}\right)$, so $x=\mu\left(z_{i}\right)$ for some $i \in I$ and such a $z_{i}$ is a preimage of $y$ by $\mu$ which belongs to $B \cup D^{B}$.

Lemma 50. Let $g \in D^{B}$ such that $\mu(g)$ is join-reducible in $L$. There exists $h \in D^{B}$ such that $\mu(h) \in \mathcal{J}_{\mathrm{c}}(L)$ and $\mu(g)=\bigvee \mu(\delta(g, h)) \vee \mu(h)$; moreover, $\mu(h)$ is non-joinprime whenever $L$ is not a Boolean algebra.

Proof. Write $\mu(g)=\bigvee \alpha$ with $\alpha \subseteq \mathcal{J}_{\mathrm{c}}(L)$ and $\alpha$ minimal with these two properties. We have then $g \leq \bigvee i(\alpha)$ so $\delta(g, h) \cup\{h\} \ll i(\alpha)$ for some $h \in D^{B}$. We have then $\mu(\delta(g, h)) \cup\{\mu(h)\} \ll \alpha$ and this relation implies that $\mu(\delta(g, h)) \cup\{\mu(h)\} \subseteq \alpha$. Indeed, since $i$ preserves the least element, $\mu(x)=\perp$ implies $x=\perp$. Thus every element of $\mu(\delta(g, h)) \cup\{\mu(h)\}$ is distinct from $\perp$ and below an atom in $\alpha$, so it is necessarily equal to such an atom. In particular, we have $\mu(h) \in \mathcal{J}_{\mathrm{c}}(L)$.

We also have $\bigvee \alpha \leq \mu(g) \leq \bigvee \mu(\delta(g, h)) \vee \mu(h) \leq \bigvee \alpha$, so $\mu(g)=\bigvee \mu(\delta(g, h)) \vee$ $\mu(h)$. By minimality, it follows $\alpha=\mu(\delta(g, h)) \cup\{\mu(h)\}$.

Suppose that $L$ is not a Boolean algebra, so we can find an atom $a \in \mathcal{J}_{c}(L)$ which is non-join-prime. Pick $f \in D^{B}$ such that $\mu(f)=a$. Observe that every element 
$\delta(f, g) \cup \delta(g, h)$ is join-prime, so every element of $\mu(\delta(f, g) \cup \delta(g, h))$ is also joinprime. If $\mu(h)$ is join-prime, then we deduce $a \leq \bigvee \mu(\delta(f, g)) \vee \bigvee \mu(\delta(g, h)) \vee \mu(h)$, so the non-join-prime $a$ has a join-cover all made of join-prime elements. Since $L$ is in the variety generated by the relational lattices, this contradicts Lemma 7 .

Let $A$ be the set of atoms of $L$ that are join-prime. While $\left(D^{B}, \delta\right)$ is a space over $P(B)$, we need to transform $D^{B}$ into a space over $P(A)$. To this end, we define a $P(A)$-valued distance $\delta_{A}$ on $D^{B}$ by

$$
\delta_{A}(f, g):=\{\mu(b) \mid b \in \delta(f, g)\} .
$$

Because of Lemma 48, we have $\delta_{A}(f, g) \subseteq A$.

Proposition 51. $\left(D^{B}, \delta_{A}\right)$ is a pairwise complete ultrametric space over $P(A)$.

Proof. $\delta_{A}$ satisfies the properties defining a distance (including being reduced and symmetric), mainly because the direct image of any function (here of $\mu$ ) preserves unions.

For pairwise completeness, observe that if $\delta_{A}(f, g) \subseteq \alpha_{0} \cup \alpha_{1}$, then $\delta(f, g) \subseteq$ $\beta_{0} \cup \beta_{1}$, where $\beta_{i}:=\left\{b \in B \mid \mu(b) \in \alpha_{i}\right\}, i=0,1$. Taking $h$ such that $\delta(f, h) \subseteq \beta_{0}$ and $\delta(h, g) \subseteq \beta_{1}$, we obtain $\delta_{A}(f, h) \subseteq \alpha_{0}$ and $\delta_{A}(h, g) \subseteq \alpha_{1}$.

We define next $v: D^{B} \longrightarrow P(A)$ by letting

$$
v(f):=\{a \in A \mid a \leq \mu(f)\} .
$$

Lemma 52. $v: D^{B} \longrightarrow P(A)$ is a module on $\left(D^{B}, \delta_{A}\right)$. That is, the relation

$$
v(f) \subseteq \delta_{A}(f, g) \cup v(g) .
$$

holds.

Proof. Suppose that $a \in v(f)$ and $a \notin \delta_{A}(f, g)$. This means that $a \leq \mu(f)$ but $b \notin \delta(f, g)$ whenever $\mu(b)=a$. Recall that if $b \in B$, then $b$ is join-prime, whence $\mu(b)$ is join-prime as well. Thus if $a \in A$ and $a \leq \mu(b)$, then $a=\mu(b)$, since we are assuming that $L$ is atomistic. Since $a \leq \mu(f) \leq \bigvee_{b \in \delta(f, g)} \mu(b) \vee \mu(g)$, $a$ is join-prime, $a \leq \mu(b)$ implies $a=\mu(b)$, we necessarily have $a \leq \mu(g)$, so $a \in v(g)$.

Lemma 53. The map $v: D^{B} \longrightarrow P(A)$ is a module on $\left(D^{B}, \delta_{A}\right)$. Moreover $v(f)=\emptyset$ if and only if $\mu(f) \in \mathcal{J}_{\mathrm{c}}(L) \backslash A$.

Proof. Suppose that $\mu(f) \in \mathcal{J}_{\mathrm{c}}(L) \backslash A$. If $v(f) \neq \emptyset$, then let $a \in A$ with $a \leq \mu(f)$. Since we are assuming that $\mu(f)$ is join-irreducible and that $L$ is atomistic, we deduce $\mu(f)=a \in A$, a contradiction.

Conversely, suppose that $v(f)=\emptyset$. This immediately gives $\mu(f) \notin A$. By the way of contradiction, suppose now that $\mu(f)$ is reducible, so use Lemma 50 to find $h \in D^{B}$ such that $\mu(h) \in \mathcal{J}_{\mathrm{c}}(L)$ and $\mu(f)=\bigvee \mu(\delta(f, h)) \vee \mu(h)$. Since $\mu(h)$ is join-irreducible, then $h \neq f$ and $\delta(f, h) \neq \emptyset$. Pick $b \in \delta(f, h)$, then $\mu(b) \in A$ and $\mu(b) \leq \mu(f)$. This gives $\mu(b) \in v(f)$, so $v(f) \neq \emptyset$, a contradiction.

Using Lemmas 35 and 53, we derive:

Corollary 54. The subspace

$$
F_{0}:=\left\{f \in D^{B} \mid \mu(f) \in \mathcal{J}_{\mathrm{c}}(L) \backslash A\right\}
$$

of $D^{B}$ is pairwise complete. 
Proof. By Lemma 53, $f \in F_{0}$ if and only if $v(f)=\emptyset$. Since $v$ is a module, the set $\left\{f \in D^{B} \mid v(f)=\emptyset\right\}$ is, by Lemma 35, a pairwise complete metric space over $P(A)$.

The following Proposition, which ends the study of bound-preserving lattice embeddings into relational lattices, shows that modulo the shift of the codomain to the lattice of a universal product frame, such a lattice embedding can always be normalized, meaning that join-irreducible elements are sent to join-irreducible elements by the left adjoint.

Proposition 55. Let $L$ be a finite atomistic lattice and let $A$ be the set of its joinprime elements. If $L$ is not a Boolean algebra and $i: L \longrightarrow \mathrm{R}(D, B)$ is a boundpreserving lattice embedding, then there exists a pairwise complete ultrametric space $\left(F_{0}, \delta\right)$ over $P(A)$ and a bound-preserving lattice embedding $j: L \longrightarrow \mathcal{L}\left(F_{0}, \delta_{A}\right)$ whose left adjoint $\nu$ satisfies the following condition: for each $k \in A \cup F_{0}$, if $k \in A$ then $\nu(k)=k$ and, otherwise, $\nu(k) \in \mathcal{J}_{\mathrm{c}}(L) \backslash A$.

Proof. Let $\left(F_{0}, \delta_{A}\right)$ be the pairwise complete space over $P(A)$ as defined in Corollary [54. By the definition of $F_{0}$, the restriction of $\mu$ to $F_{0}$ takes values in $\mathcal{J}_{\mathrm{c}}(L) \backslash A$. Therefore we can define $\nu: A \cup F_{0} \longrightarrow \mathcal{J}_{\mathrm{c}}(L)$ as follows:

$$
\nu(k):= \begin{cases}k, & k \in A \\ \mu(k), & k \in F_{0} .\end{cases}
$$

We notice next that $\nu$ is surjective. If $x \in \mathcal{J}_{c}(L) \backslash A$, then by Lemma 49, there is $y \in B \cup D^{B}$ such that $\mu(y)=x$. By Lemma 48, $y \notin B$, so $y \in D^{B}$. Since $\mu(y)=x \in \mathcal{J}_{\mathrm{c}}(L) \backslash A$, then $y$ belongs to $F_{0}$.

Let $j: L \longrightarrow \mathcal{L}\left(F_{0}, \delta_{A}\right)$ be the function defined by

$$
j(l):=\left\{x \in A \cup F_{0} \mid \nu(x) \leq l\right\} .
$$

Let us argue, in the order, that

(1) for each $l \in L, j(l)$ is a closed subset of $A \cup F_{0}$,

(2) $j$ is injective,

(3) $j$ preserves meets and (4) it preserves joins.

(1). Let $f, g \in F_{0}$ and suppose that $\delta_{A}(f, g) \cup\{g\} \subseteq j(l)$. This condition means that $\nu\left(\delta_{A}(f, g)\right)=\delta_{A}(f, g)=\mu(\delta(f, g)) \ll\{l\}$ and $\mu(g) \leq l$; it follows that $\nu(f)=\mu(f) \leq \bigvee \mu(\delta(f, g)) \vee \mu(g) \leq l$, so $f \in j(l)$.

(2). We have $j\left(l_{0}\right)=j\left(l_{1}\right)$ if and only if, for all $x \in A \cup F_{0}$, the condition $\nu(x) \leq l_{0}$ is equivalent to $\nu(x) \leq l_{1}$. As $\nu$ is surjective, this means that $l_{0}$ and $l_{1}$ have the same atoms below them, thus that they are equal.

(3). It is easily verified that

$$
j(\top)=A \cup F_{0}, \quad \text { and } \quad j\left(l_{0} \wedge l_{1}\right)=j\left(l_{0}\right) \cap j\left(l_{1}\right) .
$$

In particular, $j$ is order-preserving.

(4). Since $j$ is order-preserving, we only need to show that $j\left(l_{0} \vee l_{1}\right) \leq j\left(l_{0}\right) \vee j\left(l_{1}\right)$. To this end, we suppose that $x \in A \cup F_{0}$ is such that $x \in j\left(l_{0} \vee l_{1}\right)$, so $\nu(x) \leq l_{0} \vee l_{1}$. If $x \in A$, then $\nu(x)=x \leq l_{0} \vee l_{1}$, and since $x$ is join-prime, this gives $\nu(x)=x \leq l_{i}$ for some $i \in\{0,1\}$. This immediately yields $x \in j\left(l_{i}\right) \leq j\left(l_{0}\right) \vee j\left(l_{1}\right)$.

Suppose now that $x=f \in F_{0}$ so $\mu(f)=\nu(f) \leq l_{0} \vee l_{1}$. We have, therefore, $f \leq i\left(l_{0}\right) \vee i\left(l_{1}\right)$, so $f \triangleleft_{\mathrm{m}} \delta(f, g) \cup\{g\} \ll\left\{i\left(l_{0}\right), i\left(l_{1}\right)\right\}$ for some $g \in D^{B}$. We 
can use now Lemma 50 to pick $h \in D^{B}$ with $\mu(h) \in \mathcal{J}_{\mathrm{c}}(L) \backslash A$ (so $h \in F_{0}$ ) and $\mu(g)=\bigvee \mu(\delta(g, h)) \vee \mu(h)$.

We have then that $\mu(\delta(f, h)) \cup\{\mu(h)\} \subseteq \mu(\delta(f, g) \cup \delta(g, h)) \cup\{\mu(h)\} \ll\left\{l_{0}, l_{1}\right\}$. This relation yields

$$
\nu\left(\delta_{A}(f, h)\right) \cup\{\nu(h)\} \ll\left\{l_{0}, l_{1}\right\}
$$

or, said otherwise,

$$
\delta_{A}(f, h) \cup\{h\} \ll\left\{j\left(l_{0}\right), j\left(l_{1}\right)\right\} .
$$

This implies that $f \in j\left(l_{0}\right) \vee j\left(l_{1}\right)$.

Let us argue that $j$ preserves the least element. If $x \in j(\perp)$, then $\nu(x) \leq \perp$. We cannot have $x \in B$, so $x=f \in F_{0}$. Then $\mu(f) \leq \perp$ and $f \in i(\perp)$, contradicting the assumption that $i$ preserves bounds.

Finally, let us observe that the left adjoint of $j$ agrees, on join-irreducible elements, with $\nu$. Indeed, for each $x \in A \cup F_{0}$, we have $\nu(x) \leq y$ iff $x \in j(y)$, iff $x \leq j(y)$, where we identify, as usual, a singleton with its only element.

We conclude next the proof of the main result of this Section, Theorem 47 .

Proof of Theorem 47 . Since $\mathfrak{F}$ is rooted and full, $\mathrm{L}(\mathfrak{F})$ is a finite atomistic subdirectlyirreducible lattice by Proposition 23. Therefore, if $i: \mathrm{L}(\mathfrak{F}) \longrightarrow \mathrm{R}(D, B)$ is a lattice embedding, then we can assume, using Theorem 43, that $i$ preserves the bounds. Also, if $L(\mathfrak{F})$ is a Boolean algebra, then it is the two elements Boolean algebra, since we are assuming that $\mathrm{L}(\mathfrak{F})$ is subdirectly-irreducible. But then, $\mathfrak{F}$ is a singleton, and the statement of the Theorem trivially holds in this case.

We can therefore assume that $\mathrm{L}(\mathfrak{F})$ is not a Boolean algebra. Let us recall that $A$ is the set of join-prime elements of $\mathrm{L}(\mathfrak{F})$, see Proposition 22, According to in Proposition 55, let $\left(F_{0}, \delta_{A}\right)$ be the pairwise complete space over $P(A)$ and let $j: \mathrm{L}(\mathfrak{F}) \longrightarrow \mathcal{L}\left(F_{0}, \delta_{A}\right)$ be the lattice morphism with the properties stated there; let $\nu$ be the left adjoint to $j$. Using Corollary 41, we can also assume that $\mathcal{L}\left(F_{0}, \delta_{A}\right)=\mathrm{L}(\mathfrak{U})$ for some universal $\mathrm{S} 5^{A}$-product frame $\mathfrak{U}$.

To avoid confusions, we depart from now on from the convention of identifying singletons with their elements. We define $\psi: X_{\mathfrak{U}} \longrightarrow X_{\mathfrak{F}}$ by saying that $\psi(x)=y$ when $\nu(\{x\})=\{y\}$. This is well defined since in $\mathrm{L}(\mathfrak{U})$ (respectively $\mathrm{L}(\mathfrak{F})$ ) the nonjoin-prime join-irreducible-elements are the singletons $\{x\}$ with $x \in X_{\mathfrak{F}_{\mathfrak{U}}}$ (resp. $\left.x \in X_{\mathfrak{F}}\right)$; moreover, we have $X_{\mathfrak{U}}=F_{0}$ and each singleton $\{x\}$ with $x \in F_{0}$ is sent by $\nu$ to a singleton $\{y\} \in \mathcal{J}_{\mathrm{c}}(\mathrm{L}(\mathfrak{F})) \backslash\{\{a\} \mid a \in A\}=\left\{\{x\} \mid x \in X_{\mathfrak{F}}\right\}$. The function $\psi$ is surjective since every non-join-prime atom $\{x\}$ in $\mathrm{L}(\mathfrak{F})$ has a preimage by $\nu$ an atom $\{y\}$ and such a preimage cannot be join-prime, so $y \in X_{\mathfrak{U}}$.

We are left to argue that $\psi$ is a $p$-morphism. To this end, let us remark that, for each $a \in A$ and $x, y \in X_{\mathfrak{F}}$ (or $x, y \in X_{\mathfrak{U}}$ ), the relation $x R_{a} y$ holds exactly when there is an $\{a\}$-path from $x$ to $y$, i.e. when $\{x\} \subseteq \overline{\{a, y\}}=\{a\} \vee\{y\}$ (we need here that $\mathfrak{F}$ and $\mathfrak{U}$ are $\mathrm{S} 4$ frames).

Thus, let $x, y \in X_{\mathfrak{U}}$ be such that $x R_{a} y$. Then $\{x\} \subseteq\{a\} \vee\{y\}$ and $\nu(\{x\}) \subseteq$ $\nu(\{a\}) \vee \nu(\{y\})=\{a\} \vee \nu(\{y\})$. We have therefore $\psi(x) R_{a} \psi(y)$. Conversely, let $x \in X_{\mathfrak{U}}$ and $z \in X_{\mathfrak{F}}$ be such that $\psi(x) R_{a} z$. We have therefore $\nu(\{x\}) \subseteq\{a\} \vee\{z\}$, 
whence, by adjointness,

$$
\begin{aligned}
\{x\} \subseteq j(\{a\} \vee\{z\}) & =j(\{a\}) \vee j(\{z\}) \\
& =\{a\} \vee\{y \mid \nu(\{y\})=\{z\}\} \\
& =\frac{\{a\} \cup\{y \mid \nu(\{y\})=\{z\}\}}{}
\end{aligned}
$$

But this means that there is some $y \in X_{\mathfrak{U}}$ with $\psi(y)=z$ and a $\{a\}$-path from $x$ to $y$. But then, we also have $x R_{a} y$.

\section{REFERENCES}

[1] N. Ackerman. Completeness in generalized ultrametric spaces. p-Adic Numbers Ultrametric Anal. Appl., 5(2):89-105, 2013.

[2] K. Adaricheva. On the prevariety of perfect lattices. Algebra universalis, 65(1):21-39, 2011.

[3] S. Burris and H. Sankappanavar. A Course in Universal Algebra. Dover Publications, Incorporated, 2012.

[4] E. F. Codd. A relational model of data for large shared data banks. Commun. ACM, 13(6):377-387, June 1970.

[5] B. A. Davey and H. A. Priestley. Introduction to Lattices and Order. Cambridge University Press, New York, 2002.

[6] J. M. Dunn, M. Gehrke, and A. Palmigiano. Canonical extensions and relational completeness of some substructural logics. Journal of Symbolic Logic, 70(3):713-740, 0092005.

[7] T. Evans. Embeddability and the word problem. The journal of the London Mathematical Society, 28:76-80, 1953

[8] R. Freese, J. Ježek, and J. Nation. Free lattices. Providence, RI: American Mathematical Society, 1995.

[9] G. Grätzer. General Lattice Theory. Birkhäuser Verlag, Basel, 1998. New appendices by the author with B. A. Davey, R. Freese, B. Ganter, M. Greferath, P. Jipsen, H. A. Priestley, H. Rose, E. T. Schmidt, S. E. Schmidt, F. Wehrung and R. Wille.

[10] R. Hammack, W. Imrich, and S. Klavzar. Handbook of Product Graphs. CRC Press, Inc., Boca Raton, FL, USA, 2nd edition, 2011.

[11] R. Hirsch and I. Hodkinson. Representability is not decidable for finite relation algebras. Trans. Amer. Math. Soc., 353:1403-1425, 2001.

[12] R. Hirsch, I. Hodkinson, and A. Kurucz. On modal logics between $\mathrm{K} \times \mathrm{K} \times \mathrm{K}$ and $\mathrm{S} 5 \times \mathrm{S} 5 \times \mathrm{S} 5$. The Journal of Symbolic Logic, 67:221-234, 32002.

[13] R. Hirsch and M. Jackson. Undecidability of representability as binary relations. J. Symbolic Logic, 77(4):1211-1244, 122012.

[14] A. Kurucz. Combining modal logics. In J. V. B. Patrick Blackburn and F. Wolter, editors, Handbook of Modal Logic, volume 3 of Studies in Logic and Practical Reasoning, pages 869 - 924. Elsevier, 2007.

[15] F. W. Lawvere. Metric spaces, generalized logic and closed categories. Rendiconti del Seminario Matematico e Fisico di Milano, XLIII:135-166, 1973.

[16] T. Litak, S. Mikuls, and J. Hidders. Relational lattices: From databases to universal algebra. Journal of Logical and Algebraic Methods in Programming, 85(4):540 - 573, 2016.

[17] R. Maddux. The equational theory of $C A_{3}$ is undecidable. The Journal of Symbolic Logic, 45(2):311-316, 1980

[18] R. Maddux. Relation Algebras, volume 150 of Studies in logic and the foundations of mathematics. Elsevier, 2006.

[19] J. B. Nation. An approach to lattice varieties of finite height. Algebra Universalis, 27(4):521543, 1990.

[20] S. Priess-Crampe and P. Ribemboim. Equivalence relations and spherically complete ultrametric spaces. C. R. Acad. Sci. Paris, 320(1):1187-1192, 1995.

[21] G. Sambin. Subdirectly irreducible modal algebras and initial frames. Studia Logica, 62:269$282,1999$.

[22] L. Santocanale. A duality for finite lattices. Preprint, available from http://hal.archives-ouvertes.fr/hal-00432113 Sept. 2009. 
[23] L. Santocanale. Relational lattices via duality. In I. Hasuo, editor, Coalgebraic Methods in Computer Science, CMCS 2016, volume 9608 of Lecture Notes in Computer Science, pages 195-215. Springer, 2016.

[24] L. Santocanale and F. Wehrung. Varieties of lattices with geometric descriptions. Order, 30(1):13-38, 2013.

[25] M. Spight and V. Tropashko. Relational lattice axioms. Preprint available at http://arxiv.org/abs/0807.3795, 2008.

[26] V. Tropashko. Relational algebra as non-distributive lattice. Preprint, available at http://arxiv.org/abs/cs/0501053, 2006.

[27] F. Wehrung. Sublattices of complete lattices with continuity conditions. Algebra Universalis, 53, no. 2-3:149-173, 2005.

Luigi Santocanale, LiF, CNRS UMR 7279, Aix-Marseille Université

E-mail address: luigi.santocanale@lif.univ-mrs.fr 